\title{
LA SUBORDINATION DE L'APPRENTI A TRAVERS LES BREVETS D'APPRENTISSAGE EN ROUSSILLON À LA FIN DE L'ANCIEN RÉGIME
}

\author{
Christophe JUHEL
}

"On aura parfaitement déterminé en deux mots la nature du régime légal de l'apprentissage dans l'ancienne France, en disant qu'il y était une sorte de servitude temporaire, née des idées de monopole en matière de droit au travail, imaginée pour le plus grand profit des maitres et du renom de la fabrication nationale ou locale, et imposée à tout candidat au travail corporatif comme un premier stage d'initiation légale, comme un noviciat. Mais, ce faisant, on ne se sera encore fait aucune idée des rapports juridiques que créait ce contrat " ${ }^{1}$ "car l'apprentissage dans l'ancienne France supposait une sorte d'adoption momentanée de l'apprenti par le maître ; ce n'était pas [...] simple rapport d'ouvrier à patron, mais organisation professionnelle sur une base familiale $»^{2}$.

Tout chercheur quelque peu aguerri a vite fait de reconnaître les actes que les notaires rédigent habituellement au XVIII ${ }^{e}$ siècle. La typologie des actes notariés est assez facile à établir : contrats de mariage, testaments, quittances, ventes, louages, baux à croît ou gazailles, délibérations de confréries, corps de métiers et assemblées d'habitants en tous genres... Parmi ces actes figurent les fameux brevets $^{3} \mathrm{~d}^{\prime}$ apprentissage ${ }^{4}$. Ils sont facilement identifiables en ce qu'ils présentent une grande homogénéité formelle. Les notaires roussillonnais structurent en effet ces contrats selon un même schéma et y reproduisent des formulations similaires.

$\mathbf{1}$ - J. DASSONVILE, Le contrat d'apprentissage et son régime légal, th., dr. Lille, 1904, p. 38.

2 - J. De BONNE, L'apprentissage à Toulouse, th., droit, Toulouse, 1908, $130 \mathrm{p}$.

3 - L'usage de ce terme est avéré dans les actes notariés à partir du XVe siècle par Jules Dassonville. J. DASSONVILLE, op. cit., p. 40.

4 - A ce jour aucune étude d'envergure ne leur a été consacrée pour la province roussillonnaise. II n'existe que des travaux portant sur les métiers: A. DrapÉ, Recherches sur les corps d'arts et métiers en Roussillon sous l'Ancien Régime, Librairie nouvelle de droit et de jurisprudence Arthur Rousseau, Paris, 1898, 260 p. ; J. Lugand, Peintres et doreurs en Roussillon aux XVIle et XVIlle siècles, Trabucaire, Canet, 2006, 265 p. 
Cette ressemblance saute aux yeux lorsque l'on se cantonne à un seul notaire qui utilise la même trame pour rédiger ses actes, la même formule. Elle s'estompe cependant dès lors que l'on élargit le panel de notaires à toute la province roussillonnaise et que le nombre de brevets augmente. On se rend alors compte de la variabilité des modalités contractuelles : le vocabulaire est certes similaire mais les notaires ne recopient pas mécaniquement des formules d'actes identiques. Bien au contraire, à partir de leurs formules, ils adaptent les stipulations contractuelles aux situations qui leur sont soumises ${ }^{5}$, ne semblant pas être entravés par les statuts professionnels de la province ${ }^{6}$.

De 1779 à 1789, nous avons relevé 404 brevets d'apprentissage ${ }^{7}$ de jeunes garçons $^{8}$ rédigés par les notaires ${ }^{9}$ de la province de Roussillon. Ils témoignent de l'intense activité artisanale et commerciale de la province à la veille de la Révolution et démontrent que l'organisation professionnelle d'Ancien Régime ${ }^{10}$, tant décriée ensuite, mérite d'être réhabilitée pour ce qui regarde la formation pro-

5 - A cet égard il faut souligner la souplesse offerte par les statuts des corps de métier. Voir J. DE BonNE, op. cit., p. 36-37.

6 - On ne trouve presqu'aucune mention renvoyant aux corps de métier dans les brevets, si ce n'est lorsque des taxes spécifiques sont perçues sur les apprentis au profit de la communauté professionnelle. Les apprentis perruquiers payent un droit de chapelle, et parfois un droit de greffier du corps. Ainsi, selon le brevet du 22 octobre 1782, François Rancier qui entre en apprentissage de perruquier doit payer vingt livres de droit de chapelle et trois livres pour le greffier du corps (Arch. Dép. des Pyrénées-Orientales, 3E 13/22, $f^{\circ} 460 r^{\circ}, n^{\circ} 299$, toutes les références ci-après sont tirées de ce fonds d'archives). Ce droit est parfois qualifié de « droit de caisse » (brevet du 28 août 1783, 3E 15/89, f 345). Les apprentis tailleurs payent un droit d'apprentissage au corps de six livres (brevet du 26 mai 1782, 3E 5/79, nं 216), les apprentis boulangers un droit d'apprentissage de six livres (brevet du 6 juin 1784, 3E 25/4, $n^{\circ} 49$ ) et les apprentis droguistes versent un droit d'apprentissage dont le montant n'est pas précisé (brevet du 31 mars 1779, 3E 13/19, n 125). De même lorsqu'une règlementation nationale impose des conditions spécifiques pour entrer en apprentissage, comme c'est le cas en matière d'imprimerie et librairie où il faut avoir étudié le grec et le latin, les brevets y font référence. Voir les brevets des 19 août 1783 et 28 octobre 1788 (3E 15/89, f 333 et 3E 15/98, f $f^{\circ} 297$ ). A propos de l'application de cette règlementation en Roussillon, voir C. LENTZ, « Les gens du livre en Roussillon au XVIIle siècle : de la réception dans une communauté à la pratique des métiers du livre », in G. LARGUIER (dir.), Métiers et gens de métiers en Roussillon et en Languedoc: XVIle-XVIIle siècles, Presses universitaires de Perpignan, 2009, p. 61-81.

7 - A ce chiffre, il faudrait ajouter les brevets contenus dans les registres non communicables et ceux dont aucune copie n'a été conservée aux archives mais dont l'existence est avérée par les registres des insinuations de la série 2C. II semble cependant suffisant pour dresser quelques constats et formuler moins d'hypothèses que les auteurs qui par le passé ont envisagé la question à travers les statuts des corps et communautés de métier et trop peu de contrats d'apprentissage, ce que déplorait Roger Aubenas. R. AUBENAS, Cours dhistoire du droit privé lanciens pays de droit écrit), t. $\vee$, Contrats et obligations d'après les actes de la pratique, Aix-en-Provence, 1956, p. 90. Depuis cette époque de nouvelles recherches ont été menées, répondant pour certaines aux vœux du professeur Aubenas, voir A.-M. CoculA, « Contrats d'apprentissage à Langon et autour de Langon dans la seconde moitié du XVIII siècle », Fédération historique du Sud-ouest, Périgueux, 1971, p. 107-124 (389 contrats) ; P. BUTTEL, La croissance commerciale bordelaise dans la seconde moitié du XVIII siècle, 1973, 1164 p. (385 contrats) ; A. DeGAGE, « Les contrats d'apprentissage à Sète de 1716 à 1816 », Etudes sur Pézenas et IHérault, n 3, 1979, p. 21-35 (508 contrats) ; B. BIĖGUN, L'apprentissage au XVIII siècle dans la Sénéchaussée de Montde-Marsan, Bordeaux III, 1985 (528 contrats); N. MATHELIN, L'apprentissage à La Rochelle dans la seconde moitié du XVIII siècle 1750-1789, Bordeaux III, 1986, (468 contrats) ; B. GALLINATO, Les corporations à Bordeaux à la fin de l'Ancien Régime : vie et mort d'un mode d'organisation du travail, Presses universitaires de Bordeaux, 1992, 374 p. (522 contrats). 8 - En Roussillon, aucune formation professionnelle féminine ne semble faire l'objet de brevet d'apprentissage. II n'existe d'ailleurs pas de corps de métier féminin dans cette province.

9 - Cette pratique notariale est très précieuse. N. PELLEGRIN, «L'apprentissage ou l'écriture de l'oralité. Quelques remarques introductives », RHMC, t. 40, 1993, p. 356-386.

10 - Sur les corporations, voir E. MARTIN SAINT-LÉON, Histoire des corporations de métiers, PUF, 1941, 574 p. ; E. COORNAERT, Les corporations en France avant 1789, Paris, 6e éd., 1941 ; F. OLIVIER-MARTIN, L'organisation corporative de la France d'Ancien Régime, Paris, 1938. 
fessionnelle ${ }^{11}$. Il est vrai que l'encadrement juridique de l'apprentissage, assurant la mainmise des maitres sur la formation professionnelle, jointe au contrôle de l'accès à la maîtrise, a pu être un moyen de limiter la concurrence et de préserver au mieux le monopole corporatif sur l'artisanat et le commerce. Les brevets d'apprentissage ont même été parfois détournés de leur vocation première pour obtenir une main-d'œuvre bon marché ${ }^{12}$ mais la pratique révèle peu de situations propres à de tels soupçons ${ }^{13}$. Bien au contraire, la "légende noire " de l'apprentissage est aujourd'hui démentie car la complémentarité des obligations pesant sur les maîtres et apprentis semble démentir toute possibilité d'abus de puissance économique ou de faiblesse de la part des maîtres vis-à-vis des apprentis. L'apprentissage, rendu obligatoire dans la province en $1493^{14}$, parait avoir permis de dispenser la formation professionnelle obligatoire pour exercer un art ou une profession et espérer accéder un jour à la maîtrise. Le contrat ou brevet d'apprentissage étant la "traduction juridique de la volonté du maître et de l'apprenti de travailler ensemble $»^{15}$ à cette fin.

De l'analyse de ces brevets il ressort que la cohabitation du maitre et de son apprenti tend à humaniser leurs relations, qui dépassent ainsi le seul cadre professionnel (I), ce qui révèle la convergence de leurs intérêts et initie conséquemment une forme de solidarité entre eux (II).

\section{Une cohabitation humanisant les relations maître-apprenti}

Les notaires consacrent nombre de clauses aux conditions dans lesquelles le maître et l'apprenti doivent résider sous le même toit (A) en insistant tout particulièrement sur l'interdiction pour l'apprenti de s'absenter de chez son maitre (B).

\section{A. Des conditions de résidence commune très détaillées}

Lobligation de résider chez son maitre pour l'apprenti fait l'objet de formulations lapidaires dans les actes notariés ${ }^{16}$ tandis que l'accueil que doit réserver

11 - La formation assurée hors des corporations est plus complexe à appréhender car ne faisant pas l'objet d'actes notariés aussi visibles et clairs que les brevets. Voir C. H. CROWSTON, « L'apprentissage hors des corporations. Les formations professionnelles alternatives à Paris sous l'Ancien Régime », Annales, Histoire, Sciences sociales, 2005/2, p. 409-441.

12 - Seule la Cerdagne où sont installées des fabriques de part et d'autre de la frontière semble avoir été le théâtre d'une exploitation des apprentis. Les précautions prises par les notaires pour régler les situations conséquentes à l'éventuel départ de l'apprenti avant la fin de sa formation et les nombreuses références aux fabriques de Puycerda attestent de la concurrence existant entre les fabriques de Livia et celles de Cerdagne espagnole. Ces apprentis étaient au surplus les seuls rémunérés dans la province. En Aquitaine et à Paris, les apprentis étaient rarement rémunérés, si ce n'est lors des derniers mois d'apprentissage. Voir A.-M. CoculA, op. cit., p. 431 et S. L. KaPLAN, op. cit., p. 440.

13 - Certains brevets relèvent même de l'acte charitable. C'est le cas des brevets consentis à titre gratuit à des enfants de l'Hôpital Notre-Dame de la Miséricorde. Voir C. JuHEL, « Les brevets d'apprentissage des enfants pauvres de l'Hôpital Notre-Dame de la Miséricorde de Perpignan au XVIIIle siècle », in G. LARGUIER (dir.), Métiers... op. cit., p. 113-134. C'est le cas des brevets dérogatoires souscrits en Poitou en faveur d'un second apprenti, voir P. BoISSONNADE, Essai sur l'organisation du travail en Poitou depuis le Xle siècle jusqu â la Révolution, H. Champion, Paris, 2 t., 1900, t. 1, p. 41.

14 - Voir A. Drapé, op. cit., p. 119-124 ; E. Martin SaINT-LÉON, op. cit., p. 282-285.

15 - B. Gallinato, op. cit., p. 69.

16 - Si ce n'est pour les absences, voir infra|B. 
le maitre à son apprenti y est très détaillé car il doit être capable de recevoir en son foyer une nouvelle bouche à nourrir ${ }^{17}$. Généralement cela implique pour le maître de fournir « en sa maison les aliments lit feu et lumière audit apprenti à son égal $»^{18}$, c'est-à-dire le gîte et le couvert car tous deux doivent faire " même pot et commune habitation $»^{19}$. Ces deux dernières obligations sont fréquemment liées l'une à l'autre mais pas nécessairement. Certains brevets les distinguent.

Le logement de l'apprenti doit, selon une formule récurrente, être assuré par le maître en l'accueillant en "sa maison et boutique ${ }^{20}$ pour la durée de l'apprentissage. S'il est parfois prévu que l'apprentissage pourra être dispensé en la maison du maître " ou un tout autre endroit [où] il travaillera "21, le maître accepte de fournir au jeune impétrant "le gite dans sa maison $"^{22}$. L'obligation de loger l'apprenti est presque systématiquement complétée par la mention du feu et de la lumière dont il doit le faire bénéficier, sans davantage en préciser les modalités d'exécution. On imagine qu'elle aurait pu être quantifiée quant au bois ou aux bougies devant être mis à disposition de l'apprenti. Mais il n'en est rien : le maitre doit héberger, chauffer et éclairer son apprenti "à son égal ", ce qui en pratique signifie tout simplement qu'il doit l'accueillir comme un membre de sa propre famille ${ }^{23}$.

Assurer le couvert à l'apprenti consiste pour le maitre à lui fournir les " aliments " ou "aliments de bouche " ou encore « les aliments de manger et boire " ${ }^{24}$ "à son égal $»^{25}$, celui "de sa famille ${ }^{26}$, ou "des autres garçons $»^{27}$ " et ainsi qu'il est d'usage ${ }^{28}$. Certains actes précisent qu'il doit l'accueillir «à sa table ${ }^{29}$. Cette obligation est susceptible d'aménagements multiples, notamment lorsque l'apprentissage est long et que l'apprenti est jeune, mais pas seulement. Partager les frais au cours des premiers mois permet de réduire le prix de l'apprentissage. Un ap-

17 - F. MICHAUD-FrEjAVILLE, "Les enfants au travail, contrats d'apprentissage en Orléanais (1380-1450) », L'enfant au Moyen âge, Presses universitaires de Provence, 1980, p. 61-71.

18 - $3 \mathrm{E} 4 / 243, f^{\circ} 81$, brevet du 10 mars 1779 .

$19-3 E 22 / 258, n^{\circ} 263, f^{\circ} 634$, brevet du 2 novembre 1786

20 - Voir par exemple le brevet du 29 août 1780 (3E 40/66l, f $f^{\circ} 268$ ).

$21-3 E 21 / 463, n^{\circ} 24, f^{\circ} 44$

22 - $3 E 22 / 253, n^{\circ} 231, f^{\circ} 271$, brevet du 15 octobre 1781.

23 - Voir par exemple les brevets du 20 mai 1780 (3E 19/1062, n 46 de la « 3e main ») et du 28 avril 1782 (3E 20/399, $n^{\circ}$ 146). Cela implique que le maître doit avoir fondé une famille afin de pouvoir offrir « toutes les garanties désirables dans l'intérêt de l'enfant dont il demande à avoir la garde et à devenir l'instituteur ». II doit notamment disposer « des ressources [qui] lui permettent de nourrir et entretenir convenablement un apprenti ». E. MARTIN SAINT-LÉON, op. cit., p. 78-79.

24 - 3E 5/79, n 34l, brevet du 30 août 1782. Alain Degage cite un brevet distinguant la fourniture « du pain et du vin » que doit assumer le père de l'apprenti et « le restant de l'ordinaire ainsi qu'on le pratique avec des garçons » qui est à la charge du maitre. A. DeGAGE, op. cit., p. 28.

25 - Cette formule revient à 230 reprises dans les brevets, ce qui représente 56,93\% des contrats étudiés.

26 - La référence à la famille du maître est bien plus rare que celle du maître lui-même. Elle intervient 29 fois en complément de celle renvoyant au maître et 6 fois toute seule. Ces 35 occurrences ne représentent donc que $7,17 \%$ des brevets. $27-3 E 26 / 239, n^{\circ} 97$, brevet du 6 novembre 1785. Cette formule est récurrente pour les brevets bénéficiant aux enfants placés par l'hôpital Notre-Dame de la Miséricorde, voir C. JuHEL, op. cit., p. 125.

28 - 3 E $15 / 88, f^{\circ} 77$, brevet du 14 février 1783.

29 - 3E $14 / 59, n^{\circ} 522$ et $3 E 5 / 79, n^{\circ} 341$, brevets des 18 août 1780 et 30 août 1782. 


\section{LA SUBORDINATION DE L'APPRENTI À TRAVERS LES BREVETS...}

prenti peut initialement être nourri par ses parents avant d'être totalement pris en charge par son maître au cours des derniers mois d'apprentissage lorsqu'il est plus âgé, formé et par conséquent rentable. Ainsi Paul Montpellier, âgé de quatorze ans, placé pour quatre années en apprentissage de perruquier, est nourri et même logé par son père au cours de trois premières années, ne s'installant véritablement chez son maître que pour sa dernière année de formation ${ }^{30}$. Antoine Rolland, âgé de vingt-deux ans, doit quant à lui continuer de se nourrir chez son père pendant deux ans, ne "faisant même pot et commune habitation " avec son maître qu'au cours des six derniers mois de son apprentissage ${ }^{31}$. Pour Joseph Andreu et Nogues, la durée d'entretien partagé est encore plus réduite, fixée à trois mois à peine, sans doute parce que l'apprentissage de tonnelier est lui-même très court, ne durant que quinze mois ${ }^{32}$. Les premiers mois de formation semblent constituer une sorte de période transitoire au cours de laquelle les parents n'abandonnent pas leur enfant à un "étranger ", son maître, tandis que la fin de la formation permet à l'apprenti, désormais formé et rentable, de vivre comme les compagnons sous le toit de son maitre ${ }^{33}$.

Parfois l'entretien de l'apprenti se réduit aux seuls aliments, tout le reste demeurant à charge de ses parents ${ }^{34}$. Ainsi, tout ce qui peut concerner l'habillement de l'apprenti, c'est-à-dire "les vêtements, linge, et chaussures " ${ }^{35}$ n'est pas à la charge du maître ${ }^{36}$, cela semble " naturel et raisonnable : l'enfant arrive

30 - 3E 14/70, nं 386, brevet du 29 mai 1786

31 - $3 E$ 22/258, nं 263, f० 634, brevet du 2 novembre 1786.

32 - 3 E 25/6, n' 113 , brevet du 28 mai 1786.

33 - Cette pratique, couplée avec celle de la «période d'essai », illustre les précautions prises pour rendre ce changement de vie moins brutal pour l'apprenti et éviter d'avoir à résilier très vite le brevet. On constate en effet que les parties s'entendent parfois pour établir une période d'essai de fait lque les statuts professionnels ignorent, J. DASSONVILLE, op. cit., p. 42) en rédigeant le brevet quelques jours ou semaines après que l'apprenti ait commencé sa formation chez le maître. Ailleurs en France c'est une pratique parfois encadrée par les contrats d'apprentissage. Louis Morin donne des exemples de périodes d'essai pouvant durer plusieurs mois. Il cite le cas d'un apprenti pouvant quitter son maitre au bout de six mois en lui payant 5 livres. L. Morin, Etude sur les contrats d'apprentissage à Troyes au XVIle siècle, Troyes, 1894, 21 p., p. 14. Bernard Gallinato relève des périodes d'essai de deux à six mois. B. GaluINATO, op. cit., p. 103. Voir aussi A.-M. CoculA, op. cit., p. 430. A cet égard le brevet d'apprentissage apparaît tantôt comme « la légalisation postérieure d'accords verbaux » (F. MiCHAUD-FrĖJAVILE, «Bons et loyaux services: les contrats d'apprentissage en Orléanais (1380-1480) 》, Actes des congrès de la Société des historiens médiévistes de l'enseignement supérieur public : les entrées dans la vie. Initiations et apprentissages, $12^{e}$ congrès, Nancy, 1981, p. 203; E. MARTIN SAINT-LÉON, op. cit., p. 84 ; J. DASSONVILLE, op. cit., p. 38-40) tantôt comme un « contrat solennel, où un écrit est exigé ad solemnitatem » (J. DE BONNE, op. cit., p. 9), les actes sous seing privé étant parfois tout simplement prohibés (P. BoISSONNADE, op. cit., p. 44-45).

34 - Par le brevet du 23 août 1782, « Rose Barboteu consent et s'oblige d'entretenir pendant le présent apprentissage ledit Jacques Guria son fils de tout ce qu'il pourra avoir besoin pour sa vêture et le vivre étant a la charge dudit Ferrer de lui fournir les aliments de bouche tant seulement » (3E 30/100). A l'identique, François Prim, tailleur de La Roque, s'engage à entretenir Emmanuel Bertrand « des aliments de bouche tout seulement », tandis que la mère de ce dernier s'oblige " de l'entretenir de tout ce quil pourra avoir besoin pour sa vêture et de dormir » (3E 30/103, brevet du 24 août 1785). Enfin Raymond Ortet se charge " de nourrir dans sa maison ou habitation avec le reste de la famille [...] et à son égal sans

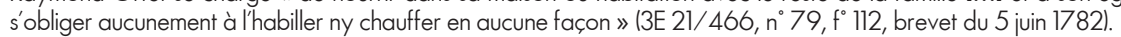

35 - 3E 22/255, n 174, f 462, brevet du 18 juillet 1783.

36 - Par exception un brevet du 14 août 1784 prévoit qu'un maître doit « de nourrir, loger, chauffer et vestir ledit apprenti à son égal » (3E 15/91, f 444). 
avec son trousseau $»^{37}$. Les brevets chargent ainsi ses père et mère, son frère aîné38, son oncle ${ }^{39}$, l'hôpital Notre-Dame de la Miséricorde ${ }^{40}$, voire l'apprenti lui-même lorsqu'il le peut ${ }^{41}$, d'y pourvoir. Joseph Grau, se plaçant en apprentissage de tailleur, paye le prix de son apprentissage fixé à cent livres, en vendant le jour de son engagement une maison pour cent dix-neuf livres, dont il retient dix livres qu'il déclare vouloir " employer à l'achat de quelque peu de linge qui lui est absolument nécessaire $~^{42}$. Il est ainsi généralement fixé par écrit qu'il reste à la charge de l'apprenti « de se fournir lui même pendant ledit tems tous les entretiens de vêture $»^{43}$. Une formule plus précise établit que le père de l'apprenti reste chargé de lui « fournir toute la robe, vêtures et chemises qui lui seront nécessaires pendant son dit apprentissage $~^{44}$. Cette clause pèche par son peu d'exactitude car tout doit dépendre de l'usure des vêtements, chaussures et linges dont se sert l'apprenti. Cependant, il ne fait aucun doute que cette obligation incombe au seul père de l'apprenti tandis que le maître ne doit en principe jamais la supporter. Un père se doit d'" entretenir ledit son fils d'habillement dans une honnette decence $"^{45}$.

Les brevets impliquant le maître dans l'habillement de l'apprenti sont donc rarissimes. Deux exceptions méritent d'être rapportées : un premier brevet partage cette tâche entre un père qui doit fournir à son fils entrant en apprentissage "les habits et chaussures nécessaires " et son maître qui doit de son côté lui fournir " un chapeau veste gilet et culotte de drap appellé vint deuxième de la couleur la plus agréable audit apprentif, une paire bas de laine et une paire de souliers le tout neuf ${ }^{46}$; et un second brevet établit qu'un maître s'oblige à acheter dans un délai de deux ans " une veste gilet et culotte de drap du pays » à son apprentii ${ }^{47}$.

Accueillir un enfant comme apprenti implique de lui fournir un lit. A ce propos, le linge de maison, accessoire au lit, n’est que rarement évoqué dans les brevets tant il semble inhérent à l'obligation de fournir un gite à l'apprenti à l'égal de son maître. Deux brevets cependant se démarquent. Le premier brevet, après avoir précisé que l'apprenti Joseph Tauriach fils "s'entretiendra d'habits et chausseures ", met également à sa charge le linge de maison qui lui est nécessaire

37 - J. DE BONNE, op. cit., p. 29

38 - 3E 44/62, nं 86, brevet du 29 avril 1781.

$39-3 E 19 / 784, f^{\circ} 18, n^{\circ} 8$, brevet du 3 janvier 1781.

40 - Lorsque l'enfant est un orphelin ou pauvre placé par cet hôpital, celui-ci lui fournit « les habits et chaussures nécessaires suivant l'uzage à l'égal des autres pauvres de la dite maison ». Voir C. JuHEL, op. cit., p. 125.

41 - Ibid.

$42-3 E 19 / 801, f^{\circ} 81, n^{\circ} 41$, brevet du 21 avril 1789

43 - 3E 30/104, brevet du 4 avril 1786

44 - 3E 30/106, brevet du 2 février 1788

$45-3 E 21 / 463, n^{\circ} 110, f^{\circ} 230$, brevet du 25 juillet 1779

46 - 3 E 26/239, nं 97, brevet du 6 novembre 1785.

47 - $3 E$ 1l/733, n' l, brevet du 26 décembre 1784. Pour Troyes, Louis Morin cite l'exemple d'une apprentie fileuse de laine et coton qui doit recevoir à la fin de ses deuxième et troisième années de formation « une chemise, une collerette et une cornette à son usage ». II évoque ensuite la pratique assez courante consistant à faire fournir un habillement à l'apprenti à la fin de sa formation par son maître. L. MoriN, op. cit., p. 13 et 19. 


\section{LA SUBORDINATION DE L'APPRENTI À TRAVERS LES BREVETS...}

en énonçant qu’il « se tiendra le lit garni de tout le nécessaire dans la maison dudit Pujarniscle ", son maître ${ }^{48}$. Cet apprenti, orphelin de père et de mère, assume sans doute ces obligations grâce à son héritage et les parents qu'il lui reste à Maureillas, d'où il est originaire et dont l'un d'eux le place en apprentissage, n'ont peut-être pas l'intention de s'en occuper ${ }^{49}$. Le second brevet met quant à lui cette obligation à la charge d'un père obligé " d'entretenir pendant ledit temps que durera ledit apprentissage ledit son fils d'habits et linge suivant son état " ${ }^{50}$ sans que l'on en devine la raison.

Par exception, lorsqu'existent des rapports dépassant le strict cadre professionnel entre un maître et son apprenti ${ }^{51}$, le premier s'engage à fournir au second des vêtements. C'est le cas de Joseph Fons qui " pour prouver audit Marty [son apprenti] l'amitié ${ }^{52}$ qu'il a pour lui a promis remettre [...] deux paires culottes chacune desdites trois années [de son apprentissage] et deux vestes dans le courant desdites mêmes années, le tout propre audit travail " ${ }^{53}$. Un autre maître s'engage à fournir à son " apprenti quand celuy cy l'exigera une paire de souliers neufs et un semelage gratis ». Il est vrai qu'ici l'apprentissage porte sur l'art de la cordonnerie $^{54}$ et que l'apprenti, majeur d'âge, se plaçant lui-même, le maître supporte entièrement son entretien, ce pour quoi il reçoit d'ailleurs un prix supérieur au tarif habituel, de l'ordre de 150 livres $^{55}$.

Cinq brevets seulement envisagent une seconde obligation, tenant à l'habillement de l'apprenti, qui pèse parfois sur le maître : le "blanchissage " ${ }^{56}$. Si quatre d'entre eux l'ajoutent simplement à la liste des obligations du maître ${ }^{57}$, le cinquième, plus tardif que les quatre autres, prévoit expressément une contrepartie financière. Cet ultime brevet, daté du 16 juin 1789, prévoit que le maître doit recevoir la somme de 48 livres pour le blanchissage et le rapiéçage des habits et

\footnotetext{
48 - 3E 44/62, n` 85, brevet du 29 avril 1781 rédigé par Me Abdon Noell et Serradell, notaire de Céret.

49 - II est placé par Estienne Llarens que le brevet qualifie in fine de «parent » lorsqu'il se porte caution solidaire des obligations souscrites par l'apprenti.

$50-3 E 21 / 472, n^{\circ} 5, f^{\circ} 4$, brevet du 4 janvier 1788. Cette clause de « prêt d'objets de literie » est fréquente à Troyes au XVIIe siècle. L. MORIN, op. cit., p. 6.

51 - A contrario, il faut souligner que lorsque ce sont les liens les plus étroits qui unissent mâtre et apprenti, c'est-à-dire ceux existant entre un père et son fils, les brevets sont beaucoup moins détaillés qu'à l'accoutumée. Il n'est pas besoin de rappeler en pareil cas ce que sont les obligations d'un père envers son fils, quand bien même il serait au surplus son maître. Deux brevets rédigés par Me Jean Mundi-Albafulla notaire à Perpignan le 27 février 1789 en faveur de deux marchands orfèvres en attestent (3E 13/51, $2^{2}$ registre, $n^{\circ} 78$ et 79$)$.

52 - Ailleurs dans le royaume cette « amitié » peut conduire à ce que l'apprentissage soit gratuit ou presque. L. MORIN, op. cit., p. 15. Dans la province de Roussillon, les brevets gratuits bénéficient seulement aux enfants pauvres et orphelins de l'hôpital Notre-Dame de la Miséricorde, voir C. JUHEL, op. cit.

$\mathbf{5 3}$ - 3E 5/80, nं 409, brevet du 30 septembre 1783.

54 - Pour les métiers du textile, Rachel Rodriguez recense parmi les soixante-quatorze brevets enregistrés à Pratsde-Mollo qu'elle a découverts pour la période 1722-1755, quinze maitres s'engageant à donner des vêtements en fin d'apprentissage. R. RodRIGUEZ, Les métiers du textile en Roussillon au XVIIle siècle, Mémoire de maîtrise, Histoire, Perpignan, 1996,55 p., p. 49.

55 - 3 E 14/60, nं 173, brevet du 13 mai 1781.

56 - Pour le Poitou, voir P. BoIsSONNADE, op. cit., p. 51, n. 1.

57 - Brevets des 15 novembre 1781 (3E 13/21, nं268), 30 septembre 1783 (3E 5/80, nं 409), 5 août 1787 (3E $40 / 707, f^{\circ} 132, n^{\circ} 102$ ) et 10 octobre 1788 (3E 26/241, $f^{\circ} 230$ ).
} 
linge de l'apprenti ${ }^{58}$. L'étendue des obligations du maître et l'arrangement portant sur le linge sont ici conséquentes au fait que l'apprenti est mineur de 25 ans, orphelin de père et de mère, et se place lui-même en apprentissage. Il n'y a, a priori, personne qui puisse s'occuper de cela parmi les membres de sa famille. Le brevet précise par ailleurs que l'apprenti s'est engagé lui-même " n'ayant ni ne voulant aucun curateur " $"$. En dehors de ces cas exceptionnels, ce sont vraisemblablement les parents de l'apprenti qui s'occupent de son blanchissage ${ }^{60}$.

Tous ces détails, intégrés dans chaque brevet d'apprentissage, attestent qu'ils sont rédigés suite à des pourparlers au cours desquels chaque obligation est soupesée afin de maintenir un équilibre propre à chaque brevet. " Rien n'est inutile dans la minutie des détails du quotidien de l'apprenti " ${ }^{61}$. L'entretien de chaque apprenti est organisé au regard des circonstances présidant à la conclusion du contrat et aux autres conditions contractuelles parmi lesquelles cette obligation doit prendre place, notamment celles de nature financière. En effet, " toute éducation professionnelle entraîne des dépenses au début mais est une source de bénéfices dans les dernières années ${ }^{62}$. Ainsi, pour les longs apprentissages, l'entretien est partagé entre les parents et le maître que ce soit dans le temps ou quant aux prestations à assumer. Aux termes du brevet souscrit par Marc Briu, " le maitre ne fournira audit son apprenti que le lit dans sa maison sauf audit apprenti de manger chez sa mère " ${ }^{63}$. Les aliments font fréquemment l'objet de cette disposition car ils constituent une charge supplémentaire pour le maitre tandis que la fourniture d'un lit ou d'une paillasse rudimentaire, n'impliquant pas de dépense répétée reste plus souvent à la charge du maitre. Ces deux types d'aménagement de l'obligation d'entretien de l'apprenti peuvent être combinés. Ainsi pour la première année d'apprentissage de François Tora, sa mère le nourrira tandis que son maitre le logera, et pour la seconde année, ce sera le maitre qui assumera pleinement l'entretien de l'apprenti ${ }^{64}$. Enfin, il arrive aussi que l'entretien demeure tout simplement à la charge des parents de l'apprenti pour toute la durée de la formation ${ }^{65}$.

$\mathbf{5 8}-3 \mathrm{E} 5 / 86, \mathrm{n}^{\circ} 255$.

59 - Ibid.

60 - J. De Bonne, op. cit., p. 29. A Sète les modalités de l'entretien sont très variées, voire « curieuses » car le frère d'un apprenti « doit blanchir toutes les semaines une chemise, un tour de col et un mouchoir » pour chacun des cinq maitres associés; un père d'apprenti, tailleur, s'engage à lui fournir « un justaucorps, sa doublure et ce qu'il convient pour le rendre parfait » et un cordonnier assure à son apprenti la « pension de barbe ». A DEGAGE, op. cit., p. 29.

61 - A.-M. COCULA, op. cit., p. 430.

62 - J. DASSONVILLE, op. cit., p. 61.

63 - 3E 14/71, n 451, brevet du 18 juillet 1786 passé devant Me François Serra, notaire à Perpignan.

64 - Selon le brevet du 24 septembre 1788, passé devant Me François Serra, notaire à Perpignan, « pendant la première année ladite Thérèse Tora nourrira son dit fils et ledit Astruc (le maître) lui donnera le lit et pendant la seconde année ledit Astruc lui fournira les aliments logement et lit vivant à sa table et à son égal » (3E 14/75, n⿳650). $65-3 E 14 / 72$, n 307, brevet du 6 mai 1787. 
Par ailleurs, le prix versé pour l'apprentissage se justifie généralement dans les contrats par les " peines et soins que prendra le maître pour enseigner son métier ». L'enseignement impliquant une perte de temps pour le maitre et une faible rentabilité de l'apprenti, il arrive que ce dernier, au cours des premiers mois de son apprentissage, rembourse ses frais d'entretien et d'enseignement, alors que pour le reste de l'apprentissage il est à l'entière charge de son maître ${ }^{66}$. Mais cela ne recouvre qu'une part de vérité car bien souvent le maitre obtient, grâce au prix, le remboursement des frais d'hébergement, de nourriture et d'entretien de son apprenti. Cela ressort notamment des brevets où l'apprenti ne verse aucun prix parce que son entretien reste à la charge de ses parents.

\section{B. Le régime des absences}

Dès lors qu'un jeune homme entre en apprentissage il doit, sauf stipulation contraire, s'installer dans la maison et boutique de son maître et ne plus la quitter. Les brevets interdisent tous à l'apprenti de s'absenter de chez son maître ; une règle qu'ils assortissent ensuite de diverses exceptions.

L'interdiction de s'absenter de chez le maître est formulée très simplement. L'apprenti doit " rester " chez son maitre ou en sa boutique. Le père ou la mère de l'apprenti s'engage parfois expressément à l'y faire rester. Ainsi Gaudérique Fabre et Vassols s'oblige à " faire rester " Pierre Fabre son fils dans la boutique de François Baux pour y apprendre le métier de cordonnier ${ }^{67}$. Si l'apprenti quitte la boutique, son père, sa mère ou toute autre personne l'ayant placé là doit normalement tenter de l'y « faire revenir ». Cette obligation ne pèse sur les responsables de l'enfant que s'il reste dans la province. Le brevet du 18 octobre 1789, plaçant Pierre Gineste chez Félix Baus maître menuisier de Perpignan, prévoit que « dans le cas où ledit Gineste fils s'absenteroit de la boutique dudit Baus pour travailler en toute autre boutique de Perpignan ou d'une de la province, ledit Gineste père fera tous ses efforts pour le faire revenir dans la boutique du même Baus, et s'il ne pouvoit y réussir en ce cas ledit Baus seroit autorisé à prendre un garçon à sa place et aux frais et dépens dudit Gineste père, ce à quoi ce dernier ne sera point tenu dans le cas où ledit son fils s'absenteroit de la province ou qu'il prit party dans le Service de Sa Majesté " ${ }^{68}$.

Le régime des absences est toujours précisément détaillé et de manière très stéréotypée. Le brevet dressé le $1^{\text {er }}$ novembre 1782 par Me Jacques Terrats, notaire à Perpignan, expose clairement la marche à suivre en la matière. A ses termes,

66 - 3E 25/6, n $n^{\circ} 113$, brevet du 28 mai 1786 passé devant Me Antoine Guiter, notaire à Perpignan.

$67-3 E 21 / 437, n^{\circ} 168, f^{\circ} 415$, brevet du 29 décembre 1782. Si le jeune garçon n'a alors que douze ans, ce n'est pas la raison déterminante de l'insertion de cette clause que le même notaire reproduit pour deux brevets où les apprentis ont seize et dix-sept ans.

$68-3 E 22 / 261, f^{\circ} 784$. 
" il ne sera point permis audit apprenti de s'absenter de chez son maître sans sa permission sous peine de rendre deux jours pour un de ceux qu'il aura manqué, si mieux n'aime ledit son maître prendre un garçon à la place et aux frais et dépends dudit apprenti, et s'il s'absente avec permission il ne rendra que jour pour jour ; de même si ledit apprenti tombe malade pendant ledit apprentissage et passe sa maladie chez son maître il rendra deux jours pour un de ceux qu'il aura manqué à la boutique en payant par ledit apprenti le médecin chirurgien et apothicaire, et s'il passe sa maladie ailleurs il rendra jour pour jour à la fin de son apprentissage $"$ "

Une règle s'impose donc : à titre exceptionnel un apprenti peut s'absenter de la boutique si son maître l'y autorise. Il s'agit là de la condition déterminante de la licéité de toute absence. Les absences licites sont celles qu'accepte le maitre, soit dans le brevet lui-même, soit au cas par cas en cours d'apprentissage. Une autorisation ou " permission expresse » est donc toujours requise. Le motif de l'absence est rarement évoqué mais quelques brevets en offrent des exemples. Certains prévoient ainsi des périodes d'absence annuelles ${ }^{70}$ allant de deux semaines à un mois pour permettre à l'apprenti d'aller faire les vendanges ${ }^{71}$, la coupe du blép ${ }^{72}$, travailler chez son père ${ }^{73}$ ou vaquer à ses obligation $s^{74}$. Un seul brevet autorise l'apprenti à s'absenter quinze jours chaque année sans avoir à se justifier mais lui impose en contrepartie de compenser cette absence en fin d'apprentissage ${ }^{75}$.

Trois autres brevets autorisent les apprentis à s'absenter pour suivre des cours. Le premier permet à un apprenti perruquier de "s'absenter chaque jour une heure et le matin et une heure le soir pour aller prendre les leçons d'écriture et de lecture ${ }^{76}$. Ce cas est unique en son genre mais on imagine qu'un perruquier doit savoir tenir un livre de comptes et que ce jeune homme de quatorze ans n'est peut-être pas encore apte à le faire. Le deuxième brevet est tout aussi facile à

\section{$69-3 E 9 / 390$.}

70 - Ce serait une nouveauté apparue dans les années 1770. A.-M. CoculA, op. cit., p. 430-431. On ne trouve cependant aucune référence religieuse dans les brevets roussillonnais alors qu'á Paris un contrat d'apprentissage de doreur permet à l'apprenti « d'aller aux instructions pour parvenir à sa première communion ». S. L. KAPLAN, « L'apprentissage au XVIIIle siècle : le cas de Paris », RHMC, t. 40, 1993, p. 436-479, p. 439.

71 - Le brevet du 31 aoôt 1789 accorde « trois semaines chaque année pendant le temps des vendanges, lequel délai ledit Tubert lui accorde pour faire son proffit ailleurs » (3E 40/671, $f^{\circ} 159$ ). Ce type d'aménagement est caractéristique des régions rurales et viticoles, ce dont témoignent les brevets bourguignons médiévaux. Voir $P$. DIDIER, « Le contrat d'apprentissage en Bourgogne aux XIVe et XVe siècles », RHDFE, 1976, p. 35-57, p. 39. Il en va de même des contrats conclus à Bordeaux. Voir B. Gallinato, op. cit., p. 84.

72 - Le brevet du 9 septembre 1782 spécifie que l'apprenti se réserve quinze jours chaque année « pour la coupe du blé propre de lui [...] ou pour qui il voudra » (3E $\left.13 / 22, f^{\circ} 403, n^{\circ} 263\right)$.

73 - Le brevet du 19 octobre 1784 accorde deux semaines par an pour « aller travailler chez son père ou voir ses parents » (3E $\left.21 / 468, n^{\circ} 133, f^{\circ} 233\right)$.

74 - Brevet du 25 février 1788 par lequel le maitre accorde « un mois pendant l'été prochain pour pouvoir vacquer aux affaires qu'il voudra » (3E 56/165).

75 - II énonce qu' « il sera cependant libre au même apprenti de s'absenter les quinze jours de chaque année qu'il jugera à propos à la charge par luy de le rendre à la fin du présent bail ». Brevet du 13 mars 1781 (3E 14/60, n 173).

76 - 3E 14/60, nं 314, brevet du 14 mai 1781. 
comprendre car l'une de ses clauses autorise un apprenti orfèvre à " prendre une heure du jour pour se perfectionner dans le dessin " ${ }^{77}$. Il est pourtant curieux que le maître orfèvre ne dispense pas lui-même cet enseignement dans la mesure où les maitres graveurs ciseleurs l'assument lorsqu'ils forment leurs apprentis ${ }^{78}$. D'ailleurs Jean Godet, graveur, s'engage à enseigner « l'art de graveur et cizelleur ainsi que le dessin, par ou ledit $S$ Hiacinthe Feret doit commencer $"{ }^{79}$. Cet ordre n'est pas envisagé dans le brevet d'apprentissage engageant le même maître envers Christophe $\mathrm{Cayrol}^{80}$. L'ordre dans lequel les divers enseignements doivent être dispensés est ainsi prédéfini. Enfin, aucun autre brevet d'apprentissage d'orfèvre ne mentionne ce type d'aménagement ${ }^{81}$. Le troisième brevet impose à Joseph Dedeu maitre en chirurgie de Prades de " permettre et obliger même ledit Dominique Salamo [son apprenti] d'aller tous les jours a l'ecole sans pouvoir demander pour raison de ce aucune indemnitét $e^{82}{ }^{83}$. Ce jeune garçon, dont l'âge est inconnu, se nomme comme son défunt père Dominique Salamo qui était lui-même maître chirurgien. Ce dernier n'ayant sans doute pas eu le temps d'assurer l'avenir de son fils de son vivant, sa veuve s'en charge en s'assurant qu'il pourra finir sa scolarité tout en se formant au métier de son père auprès d'un chirurgien ${ }^{84}$.

L'apprenti est également autorisé à quitter la boutique pour aller manger ou dormir chez ses parents lorsque le brevet le prévoit de même qu'en cas de maladie. La maladie de l'apprenti est envisagée par tous les brevets car elle l'empêche de travailler $^{85}$ et suppose donc qu'il rende des jours de travail une fois le terme de l'apprentissage échu ${ }^{86}$. Le nombre de jours "à rendre " varie selon le nombre de jours manqués mais aussi en fonction des conditions dans lesquelles l'apprenti doit être soigné. S'il passe sa maladie chez ses parents il doit rendre jour pour jour alors que s'il passe sa maladie chez son maître et que celui-ci lui fournit les bouillons ${ }^{87}$, voire les remèdes et médicaments ${ }^{88}$, l'apprenti doit rendre deux jours pour un. Les frais médicaux, qu'il s'agisse de ceux d'un médecin, chirurgien ou apothicaire, ne sont quant à eux jamais pris en charge par le maître. Un brevet

77 - 3E 13/19, nं 301, brevet du 16 septembre 1779.

78 - $3 E$ 25/5, nं 146 et $3 E 25 / 8, n^{\circ} 114$, brevets des 15 septembre 1785 et 28 août 1788 .

79 - $3 E$ E 25/5, nं 146 , brevet du 15 septembre 1785.

80 - 3E 25/8, nं 114, brevet du 28 août 1788 .

81 - Voir les deux brevets conclus le 27 février 1789 devant Me Jean Mundi-Albafulla, notaire de Perpignan (3E 13/51, 2 registre, nos 78 et 791 .

82 - A priori à Troyes au XVlle siècle, ces heures de cours à l'école sont aux frais des parents. Eł parfois, ce sont les maîtres qui prennent en charge l'instruction de l'apprenti à l'image d'une couturière qui s'engage à « enseigner autant qu'elle le pourra à lire et à écrire » à la fille d'un boulanger. L. MORIN, op. cit., p. 17. Ce type d'aménagement existe aussi à Bordeaux, B. Gallinato, op. cit., p. 87.

83 - $3 E$ 26/24l, $f^{\circ} 230$, brevet du 10 octobre 1788.

84 - Ibid.

85 - C'est un obstacle à la rentabilité de l'apprentissage. A.-M. CoCULA, op. cit., p. 430.

86 - C'est une pratique assez courante. Voir J. De BONNE, op. cit., p. 48-49.

87 - Par exception, quelques brevets laissent les bouillons à la charge de la famille de l'apprenti. Voir par exemple les brevets des 14 avril 1784 (3E 5/81, n 123) et 2 novembre 1786 (3E 22/258, n²63, f 634).

88 - Par exemple les brevets des 24 novembre 1782 (3E 36/108, n' 184) et 3 septembrel783 (3E 20/400, $n^{\circ} 277$ ). 
prévoit que si l'apprenti passe sa maladie chez son maître, aucun jour ne sera ensuite rendu mais " il sera payé à celui-ci la dépense pour raison de sa maladie comme bouillons et remèdes fournis audit apprentif $"$ ${ }^{89}$.

Quelques brevets envisagent l'absence définitive de l'apprenti, c'est-à-dire leur résiliation anticipée ${ }^{90}$, notamment en cas d'infirmité ou de décès de l'apprenti. Ainsi le brevet du 17 juin 1781 par lequel le jeune Joseph Carrere est placé chez Joseph Tore maître cordonnier pour femmes précise que si l'apprenti «par quelque événement imprévu comme par un rhumatisme et toute autre maladie ou incommodité qui l'empêcha de travailler ne pouvait pas continuer son apprentissage, alors ledit brevet d'apprentissage sera pleinement résilié, ledit Tore ne devant cependant pas rendre l'argent qu'il aurait reçu pour raison du présent apprentissage, et si à l'époque dudit événement imprévu sus précisé le même Tore n’avait pas reçu l'entière somme qui sera ci-après mentionnée le même Carrere fils sera pleinement déchargé » ${ }^{91}$. Quelles que soient les circonstances financières, aucune somme ne doit donc être versée une fois le brevet résiliép ${ }^{92}$. La même solution est retenue dans sept autres brevets, rédigés par les notaires de Saillagouse ${ }^{93}$, qui précisent que si l'apprenti quitte son maître pour " cause de mort, infirmité ou autre légitime empechement qui le priveroit visiblement de pouvoir continuer [...], en ce cas ny les uns ny les autres ne seront point tenûs de là en avant à exécuter le présent brevet d'apprentissage en rien de son contenu ${ }^{94}$.

Le décès de l'apprenti ou du maître ${ }^{95}$ mettant naturellement fin au contrat d'apprentissage ${ }^{96}$, il est parfois prévu qu'en pareil cas le père de l'apprenti ne payera le prix de l'apprentissage « que par prorata du temps que l'apprentissage

89 - 3E 29/44 f 654, brevet du 6 décembre 1789.

90 - Aucun brevet n'envisage de prolongation du temps d'apprentissage au-delà des jours dus pour compenser les absences de l'apprenti au cours de la formation. Mais ailleurs il semble que certains apprentis restent parfois bien plus longtemps au service de leur maître sans que l'on n'en connaisse la raison. F. MiCHAUD-FrÉJAVILLE, « Bons et loyaux services... op. cit., p. 205. Il arrive même que des apprentis longuement absents de leur atelier doivent recommencer le temps de leur service, voir P. BolsSONNADE, op. cit., p. 43 et 49 et B. Gallinato, op. cit., p. 98.

$91-3 \mathrm{E} 5 / 78, n^{\circ} 210$.

92 - Louis Morin mentionne un contrat d'apprentissage où l'apprenti peut quitter son maître au bout de six mois s'il le veut en payant six livres « pour toutes choses » et un autre où une mère s'est réservée la possibilité de retirer son fils à tout moment « en indemnisant le maître au prorata du temps passé chez lui ». L. MoRIN, op. cit., p. 14. Joseph De Bonne mentionne également un contrat toulousain où une mère peut reprendre son fils « s'il ne peut apprendre » en remboursant au maître ses frais de bouche. J. DE BONNE, op. cit., p. 40. En général les personnes ayant placé le garçon en apprentissage s'engagent à ne pas l'enlever de chez son maître ni à l'inciter à fuguer. P. DIDIER, op. cit., p. 41-42.

93 - Me Joseph Galard insère cette formule dans trois brevets sur les huit qu'il rédige et Me François Nicolau l'intègre systématiquement dans ses quatre brevets.

94 - Voir par exemple le brevet du 8 mai 1782 rédigé par Me Galard (3E 56/159, n¹14) et du 6 novembre 1784 rédigé par Me Nicolau (3E 56/60, n’314).

95 - C'est une règle classique, hormis le cas où « la veuve ou les héritiers prenaient la suite des affaires », ce qui maintenait l'apprenti dans sa situation. J. DASSONVILLE, op. cit., p. 57. En outre, il était possible pour une veuve et son fils de souscrire un brevet pour prendre un apprenti et le former au métier exercé par leur défunt mari et père (3E 23/46, $f^{\circ} 129$, brevet du 5 octobre 1785). Cas rarissime car il n'y a pas d'autre trace d'intervention d'une femme dans la formation professionnelle suite au décès de son mari en Roussillon alors que cela semble se pratiquer régulièrement ailleurs. E. MARTIN SAINT-LÉON, op. cit., p. 89 ; P. BOISSONNADE, op. cit., 39-40.

96 - Voir P. Didier, op.cit., p. 47 ; F. MiChaUd-FréJAVILLE, « Bons et loyaux services... op. cit., p. 183-208. 
aura duré ${ }^{97}$ ou encore qu'une mère ne sera pas tenue de payer la seconde moitié du prix ${ }^{98}$. Un brevet signé le 4 janvier 1782 prévoit que si le maître meurt " ses héritiers seront tenus de rembourser audit apprenti le prix bas spécifié a proportion du tems qui luy manquer pour finir ledit apprentissage " de même que si l'apprenti décède au cours de sa première année d'apprentissage ${ }^{99}$. Le brevet du 11 mars 1784 plaçant Marc Valls chez Bonnaventure Bernard maître tisserand à lin de Codolet, offre une alternative en cas de décès prématuré du maître puisque "les héritiers de celuy cy seront obligés de mettre ledit apprenti dans une autre boutique de tisserand pour y parachever son apprentissage ou bien de luy rendre son argent à proportion du temps ${ }^{100}$. Il semble que la vente ou cession à un autre maître se pratiquait assez facilement ailleurs dans le royaume ${ }^{101}$.

L'hypothèse d'une fuite de l'apprenti " par libertinage » est citée par dix brevets, quatre rédigés par Me Antoine Ferriol-Pastor à Millas ${ }^{102}$ et six par Me Joseph Gauze à Rivesaltes ${ }^{103}$. Ce départ ou cette absence sans motif et sans permission ouvre le droit pour le maître d'embaucher un garçon en lieu et place de l'apprenti absent ${ }^{104}$. Aucun brevet ne prévoit qu'un proche parent tel un frère ou cousin pourrait prendre sa place ${ }^{105}$.

De la même manière si l'apprenti quitte la boutique " par dégout " "106, le maitre doit en avertir celui qui a placé le jeune garçon afin qu'il tente de le convaincre de revenir ou d'en assumer le remplacement ${ }^{107}$. Une mère doit en pareil cas « le chercher, faire chercher, et le ramener s'il est possible pour finir son tems $»^{108}$. Mais aucun brevet local ne prévoit que l'apprenti doive être "appréhendé au corps en cas d'absence illégitime et ramené à son maître " ${ }^{109}$. Il importe peu que l'apprenti s'absente « pour travailler ailleurs ou pour quitter ledit métier ». Son représentant et lui sont tenus de payer un garçon pour parachever

97 - 3E 26/242 f 302, brevet du 21 septembre 1789. Voir aussi le brevet du 31 aoôt 1789 (3E 40/671, f 159).

98 - 3 E 21/463, n 24, f० 44, brevet du 28 février 1779.

$99-3 \mathrm{E} 21 / 487, \mathrm{n}^{\circ} 1$.

$100-3 E 21 / 439, n^{\circ} 30, f^{\circ} 51$. Voir en ce sens J. De BONNE, op. cit., p. 45-46.

101 - E. MARTIN SaINT-LÉon, op. cit., p. 88-89; J. DASSONVILLE, op. cit., p. 58-60.

102 - Par exemple voir le brevet du 13 mai 1779 (3E 29/34, $f^{\circ} 299$ ).

103 - Par exemple voir le brevet du 9 septembre 1783 (3E 36/109, n’ 122).

104 - Cela se pratique à Bordeaux aussi, B. GallinAto, op. cit., p. 98-100.

105 - Cela existe en Bourgogne à la fin du Moyen âge. P. DiDIER, op. cit., p. 42.

106 - Terme employé dans dix-sept brevets dont seize rédigés par Me Sauveur Jaume au profit d'enfants de la Miséricorde (voir C. JUHEL, op. cit., p. 130) et le dernier par Me François Maria, notaire à Thuir, le 6 novembre 1785 (3E 26/239, $n^{\circ}$ 97). La résiliation pour cause de dégout pouvait être évitée en aménageant une période d'essai au cours de laquelle l'apprenti pouvait jauger son intérêt pour le métier et le maître d'évaluer son aptitude.

107 - Aux XIVe et XVe siècles en Bourgogne, cette clause, qui est assez fréquente en Roussillon au XVIIIle siècle, est exceptionnelle car les parents ne sont tenus qu'à une « diligence générale et ne [répondent] pas du résultat ». P. DIDIER, op. cit., p. 42.

108 - 3E 22/255, nं $174, f^{\circ} 462$, brevet du 18 juillet 1783.

109 - «Apprentis en Forez sous l'Ancien Régime. Les contrats d'apprentissage aux XVIIe et XVIIIe siècles dans le Montbrisonnais », Cahiers de Village de Forez, 2011,74 p., p. 8. Voir également J. DASSONVILLE, op. cit., p. 54 et P. BoISSONNADE, op. cit., p. 49. Bernard Gallinato cite un contrat par lequel une femme s'engage « à faire arrêter et retenir son neveu dans les prisons de l'hôtel de ville autant que le maître le jugera à propos ». B. GaLLINATO, op. cit., p. 99. 
la période initialement fixée ${ }^{110}$, ou encore les jours lui manquant pour combler toutes ses absences ${ }^{111}$.

Peu de brevets offrent la faculté à l'apprenti de quitter son maitre quand il le désire $^{112}$. Par exception, le brevet conclu le 21 septembre 1789 devant Me Maria notaire à Thuir prévoit qu'" en cas ledit apprentif voulut quitter la maison dudit Conte [son maître] et renoncer audit métier, il devra donner audit Sr Conte une indemnité de soixante onze livres en sus prix ci-dessus convenu ${ }^{113}$. Un autre brevet, en date du 29 mars 1784 établit plus simplement que si l'apprenti "voulait quitter la boutique [de son maittre] avant l'expiration des deux années, l'argent qu'il aura payer pour comptes dudit apprentissage le devra tout au profit du même" maître ${ }^{114}$.

Parallèlement rares sont les brevets permettant à un maître de congédier un apprenti qui ne serait pas doué ${ }^{115}$, pas suffisamment diligent ou travailleur ${ }^{116}$. Avare en détails sur ce point, le brevet du 29 mars 1784 énonce que si durant les deux années d'apprentissage de Pierre Prats chez Joseph Pouig maître boulanger, ce dernier « était dans le cas de renvoyer ledit Prats pour une raison légitime, il ne veut exiger du même Prats que le prorata du temps qu'il aura resté chez lui $»^{117}$. L'expression " raison légitime " semble couvrir toute hypothèse d'incompétence de l'apprenti, voire d'infirmité ou maladie.

Quelques brevets envisagent le départ d'un apprenti causé par le maître. En effet celui-ci a l'obligation de traiter son apprenti " humainement " ${ }^{118}$, " avec humanité " ${ }^{19}$, « humainement et doucement ${ }^{120}$ comme il appartient » ${ }^{121}$, "à l'égal de sa famille "122. Il doit avoir "tous les égards et ménagements possibles " ${ }^{123}$

$\overline{110}$ - 3E 16/619, nं338, brevet du 26 novembre 1780.

111 - Voir par exemple le brevet du 28 avril 1789 (3E 10/112, $\left.f^{\circ} 172 v^{\circ}\right)$.

112 - Cette hypothèse se vérifie ailleurs. Voir B. GaLLINATO, op. cit., p. 103.

113 - Cette faculté existe ailleurs. A Troyes au XVIle siècle, un contrat prévoit que l'apprenti pourra « quitter le service s'il ne s'y trouve pas bien », un autre que la « mère conserve la faculté de retirer son fils du service en indemnisant le maitre au prorata du temps passé chez lui » et encore un autre qu'une fillette doit y apprendre le métier «si à elle ne tient et qu'elle le puisse comprendre ». L. MORIN, op. cit., p. 14 et 20.

$114-3 E 5 / 81, n^{\circ} 110$.

115 - En pareil cas en Bourgogne à la fin du Moyen âge des brevets prévoient la possibilité pour un maître de libérer un apprenti pour qu'il apprenne un métier plus adapté à ses qualités. P. DIDIER, op. cit., p. 48.

116 - C'est possible à Troyes au XVIle siècle pour un maître qui doit alors verser 12 livres à titre de dédommagement. L. Morin, op. cit., p. 14.

$117-3 E 5 / 81, n^{\circ} 110$

118 - Un terme employé à douze reprises seulement dans les brevets.

119 - 3E 12/96, n²02, brevet du 8 novembre 1785 .

120 - A la même époque ces termes sont employés en Aquitaine, à Troyes et Paris. Ces formules ne sont pas anodines à en croire les brutalités subies par les apprentis imprimeurs de la part de leurs maîtres troyens. A.-M. CocuLA, op. cit., p. 428 ; L. MorIN, op. cit., p. 17, n. 1; S. L. KaPLAN, op. cit., p. 440. Voir également DufrêNE, La misère des apprentis imprimeurs, appliquée par le détail à chaque fonction de ce pénible état, 1710 et N. CONTAT dit LE BRUN, Anecdotes typographiques où l'on voit la description des coutumes, mœurs et usages singuliers des compagnons imprimeurs..., 1762, publié par G. Barber, Oxford, 1980, 163 p.

121 - 3E 29/38, f 161 . Brevet du 17 février 1783

122 - Voir par exemple le brevet du 26 décembre 1784 (3E $\left.11 / 733, n^{\circ} 1\right)$.

123 - Expression utilisée à quatre reprises par Me Joseph-Simon Blad, notaire à Perpignan. Voir par exemple le brevet du 22 janvier 1782 (3E 22/254, $\left.n^{\circ} 17, f^{\circ} 71\right)$. 
pour son apprenti. Il doit en conséquence s'abstenir de "mauvais traitement " ${ }^{124}$. Cette obligation lui incombe ainsi qu’à " ceux de sa maison ». Cette précision n'est pas inutile car il est probable que de jeunes apprentis ont pu être tyrannisés par des maîtres, parents du maître ou compagnons, plus âgés et expérimentés qu'eux $^{125}$. Un brevet du 15 août 1779 précise à cet égard que « si ledit apprentif quitte ou est obligé de quitter a cause de mauvais traitement de la part dudit Puig [son maître] ou de ceux de sa maison, ce dernier sera obligé à refaire audit Guitart [apprenti] à proportion et par proratta du temps qui manquera pour l'expiration dudit apprentissage ». Des brutalités réitérées autorisent l'apprenti à écourter son apprentissage et imposent généralement au maître le remboursement de la somme correspondant à la période de formation non assurée. Les violences dont se rend coupable le maître lui interdisent de prendre un garçon aux frais de cet apprenti ou de sa famille ${ }^{126}$ ou de réclamer le reste des sommes dues pour l'apprentissage ${ }^{127}$. Les brevets les plus " radicaux " prévoient dans un tel cas le remboursement intégral du prix de l'apprentissage. Ainsi un brevet du 22 avril 1783 énonce que si un apprenti « est obligé de quitter la maison de son maitre par les mauvaises façons ou par la faute de ce dernier ledit [maître] sera obligé de restituer la susditte somme qui forme le prix dudit apprentissage audit [apprenti] ${ }^{128}$. Un autre brevet, plutôt pragmatique, impose au maître en pareille circonstance, c'est-à-dire de départ prématuré de l'apprenti causé par sa faute de "procurer audit [apprenti] une autre boutique pour y finir ledit terme fixé a deux ans et demi, reservant l'election de la boutique au gré dudit [apprenti] " ${ }^{129}$. De telles stipulations ne sont cependant accompagnées d'aucune mention du droit de correction dont dispose le maître sur l'apprenti ${ }^{130}$. L'autorité du maître sur l'apprenti n'est envisagée que professionnellement. Aucun brevet ne dispose par exemple que l'apprenti a été placé « au pouvoir » de son maître comme cela

124 - Cette expression est employée dans six brevets de deux notaires, Me Jean Companyo-Bombes de Céret et Me Alexis Conte de Perpignan qui ont respectivement rédigé sept et quarante-et-un brevets au cours de cette période. A Toulouse on trouve aussi l'expression « rudes traitements », J. DE BONNE, op. cit., p. 50.

125 - A.-M. CoculA, "Contrats d'apprentissage du XVIIIle siècle : quelques enseignements d'une moisson aquitaine », RHMC, t. 40, 1993, p. 423-435, p. 428.

126 - Voir par exemple le brevet du 30 août 1782 (3E 5/79, nं 341).

127 - La solution est la même qu'en cas de décès prématuré du maître ou de l'apprenti, c'est-à-dire en cours d'apprentissage. Le brevet du 28 février 1779 prévoit tous ces cas de figure. Le payement du prix devant être réalisé en deux fois, le premier versement à la signature et le second un an plus tard, il est prévu que si par la faute du maître son apprenti quitte sa boutique au cours de la première année sa mère ne versera pas la seconde moitié du prix (3E $\left.21 / 463, n^{\circ} 24, f^{\circ} 44\right)$. Cette même solution peut être adoptée chaque fois que le payement du prix de l'apprentissage est fractionné (3E 5/84, $n^{\circ} 61$, brevet du 26 février 1787).

$128-3 E 21 / 467, n^{\circ} 114, f^{\circ} 209$.

129 - 3 E 40/662, f 304 , brevet du 10 décembre 1781.

130 - E. MARTIN SAINT-LÉON, op. cit., p. 85. Le maître ne jouit plus d'une « délégation de puissance paternelle » ne bénéficiant plus de son «triple droit de surveillance, de garde et de correction», J. DASSONVILE, op. cit., p. 53. Tout au plus, ce droit de correction survit-il vis-à-vis des apprentis éloignés de leurs parents, privés alors de réels moyens de défense, sans qu'il soit besoin d'y consacrer une clause. B. GALLINATO, op. cit., p. 83. II faudrait d'ailleurs mener des recherches sur les brevets conclus en Roussillon au début du XVIIIle siècle pour vérifier si, comme cela est avéré pour l'Aquitaine, la mention du droit de correction a disparu depuis 1740. A.-M. CoculA, op. cit., p. 431. 
s'écrit en Aquitaine ${ }^{131}$. Par ailleurs, aucun brevet ne mentionne la possibilité de le résilier pour cause de "mésentente " ou "incompatibilité d'humeur " entre le maître et l'apprenti. En revanche, des actes de résiliation passés devant notaire avèrent que ce type de rupture se pratique dans la province sans que cela ne soit prévu par le brevet initial. Ainsi, l'acte dressé le 30 décembre 1780 par Me Jacques Companyo notaire à Céret énonce que Joseph Barraban se serait placé en apprentissage chez Thomas Clarimon cordonnier de Céret le 29 août précédent pour dix-huit mois mais que « lesdites parties n'ayant pû lier ensemble par des raisons a elles connûes ${ }^{132}$, déclarent dissoudre les conventions apposées dans ledit acte de brevet d'apprentissage ${ }^{133}$. De même, le 19 juin $1785^{134}$, Pierre Calmon fils d'un brassier de Rivesaltes, résilie l'apprentissage qu'il a commencé chez Antoine Joué boulanger de Saint Laurent de la Salanque six mois plus tôt ${ }^{135}$, déclarant ne pas pouvoir « travailler et continuer par l'incompatibilité d'humeur ». Enfin, le brevet conclu en début d'année 1786 pour deux ans et demi entre Etienne Savis, fils de Jean Savis brassier de Corbère, et Michel Mascle maître tailleur, est résilié le 10 avril 1787 parce que « lesdits Mascle et Etienne Savis ne pouvant simpathiser ni accorder ensemble, le dernier se seroit retiré dans la maison paternelle et auroit refusé de se rendre désormais en la boutique dudit Mascle ${ }^{136}$. Joseph De Bonne cite, quant à lui, l'exemple d'un brevet contracté en 1762 pour sept années et résilié au bout de trois seulement parce que maittre et apprenti ne peuvent « se compatir ensemble $»^{137}$.

La règle selon laquelle l'absence non autorisée de l'apprenti permet au maître de " prendre un garçon à sa place aux frais et dépens " de celui qui le place en apprentissage ${ }^{138}$ s'applique notamment lorsque l'apprenti choisit d'aller travailler dans une autre boutique. Mais il existe deux circonstances dans lesquelles rien n'est dû par l'apprenti ou son responsable. Il s'agit de l'entrée au service armé du roi ${ }^{139}$ et du départ de la province. L'engagement dans l'armée royale est envisagé par cinq des quarante-cinq notaires et trente-quatre brevets seulement, soit 8,41 $\%$ de ces actes. Antoine Guiter, notaire de Perpignan, n'évoque l'« engagement dans les troupes du roi " ou l'entrée dans "quelque régiment " que dans six des vingt-deux brevets qu'il dresse pour écarter en pareil cas les conséquences

131 - A.-M. CoCULA, op. cit., p. 429.

132 - Formule sibylline en usage à Bordeaux à la même époque. B. Galuinato, op. cit., p. 103.

133 - $3 E 40 / 661, f^{\circ} 400$

$134-3 \mathrm{E} 22 / 257, \mathrm{n}^{\circ} 156, f^{\circ} 361$.

135 - $3 E$ 22/256, nं $386, f^{\circ} 991$.

$136-3 \mathrm{E} 26 / 240$.

137 - J. De BONNE, op. cit., p. 41.

138 - C'est une règle très répandue. A. DeGAGE, op. cit., p. 27.

139 - C'est un « événement assez rare » selon Jules Dassonville. J. DASSONVILL, op. cit., p. 6l. Aucun brevet n'évoque l'entrée en religion alors que cette mention existe ailleurs dans le royaume. F. OLIVIER-MARTIN, L'órganisation corporative de la France d'Ancien Régime, Sirey, 1938, 565 p., p. 130. 
habituelles des absences injustifiées ${ }^{140}$. Jacques Companyo, notaire de Céret, ne l'envisage qu'une seule fois dans les quatre brevets qu'il rédige, en prévoyant que l'apprenti peut s'engager " au service de Sa Majesté ou de tout autre souverain ». Il est le seul à imaginer qu'un jeune homme puisse prendre le parti d'un souverain étranger ${ }^{141}$. A l'inverse, un seul notaire insère systématiquement une clause à ce propos dans ses brevets. Il s'agit de Joseph-Simon Blad qui évoque dans ses vingt brevets la possibilité que l'apprenti prenne « le parti dans le service » « de Sa Majesté " ou " du Roy ». Paul Pilas-Purxet, notaire de Vinça, prévoit quant à lui cette entrée au service du roi dans cinq de ses six brevets. Mais Pierre Philip, autre notaire de Vinça, très précautionneux lorsqu'il s’agit de déterminer le régime des absences d'Abdon Delseny au cours de son temps d'apprentissage en envisageant la possibilité qu'il puisse prendre le parti de Sa Majesté ${ }^{142}$, se montre en revanche moins précis dans le second brevet qu'il rédige au cours de cette période ${ }^{143}$.

De la même manière, rien n'est dû si l'apprenti quitte la province et disparaît ou part travailler dans une autre province. Seul Joseph-Simon Blad évoque cette possibilité dans tous ses brevets ${ }^{144}$, Guiter le prévoit à cinq reprises ${ }^{145}$ et Alexis Conte ne l'insère que dans un seul brevet ${ }^{146}$ sur les quarante-et-un qu'il rédige. Ces vingt-sept brevets ne représentent que $6,68 \%$ du total des actes. Lorsqu'elles sont prévues dans les brevets ces deux circonstances exceptionnelles constituent des dérogations au régime des absences injustifiées et résiliations car le maitre est privé de son droit de prendre un garçon à la place de l'apprenti ${ }^{147}$. Certains brevets prévoient qu'une fois l'apprenti de retour dans la province il se doit de terminer son apprentissage ${ }^{148}$. Jusqu'à cet hypothétique retour le maître est tenu d'" attendre que ledit apprenti soit revenu du service de Sa Majesté " afin que celui-ci puisse rendre " jour pour jour de ceux qu'il aura manqué » ${ }^{149}$. Le droit du maître de louer un garçon à la place de l'apprenti absent sans permission reprend vigueur au retour et désengagement de celui-ci ${ }^{150}$.

Ainsi l'entrée dans l'armée royale et le départ de la province constituent des conditions suspensives et non extinctives des obligations nées du brevet. Le retour de l'apprenti l'oblige à accomplir les prestations promises ou à dédommager le maître si le brevet le prévoit ou si les parties en conviennent. Obliger maître et

\footnotetext{
$140-3 E 25 / 6, n^{\circ} 113$ et $3 E 25 / 9, n^{\circ} 138$, brevets des 28 mai 1786 et 7 juin 1789

$141-3 E 40 / 661$, f 43 , brevet du 13 février 1780 conclu au profit de Joseph Miquel.

142 - 3E19/801, $f^{\circ} 29, n^{\circ} 15$, brevet du 31 mars 1789.

143 - 3E19/801, $f^{\circ} 81, n^{\circ} 41$, brevet du 21 avril 1789.

144 - Le brevet du 18 juillet 1783 conclu au profit de Côme Porra le prévoit. 3E 22/255, n 174, f 462.

145 - II le fait en même temps qu'il envisage un engagement dans un régiment.

$146-3 E 5 / 76, n^{\circ} 18$, brevet du 3 janvier 1780.

147 - Le brevet du 8 avril 1781 conclu au profit de Joseph Jocavell prévoit que le maître « ne pourra pas louer à sa place » (3E $\left.19 / 732, f^{\circ} 317, n^{\circ} 92\right)$.

148 - 3E 22/260, nं 131, f 298, brevet du 7 avril 1788.

149 - $3 E 19 / 801, f^{\circ} 29, n^{\circ} 15$, brevet du 31 mars 1789.

150 - 3E 19/732, f $317, n^{\circ} 92$, brevet du 8 avril 1781 .
} 
apprenti à cohabiter contre leurs grés serait stupide et néfaste à la bonne marche de l'activité ou boutique du maître. Il est préférable que tous deux soient d'accord pour poursuivre l'apprentissage et soient liés par une communauté d'intérêts.

\section{La communauté d'intérêts entre les maîtres et apprentis}

Du point de vue professionnel, les relations entre maittre et apprenti ont deux objets : ils doivent collaborer pour assurer la bonne formation de l'apprenti (A) et faire en sorte que ce dernier soit en état de travailler au profit de son maittre (B).

\section{A. Le cour du brevet : la bonne formation de l'apprenti}

Deux types de clauses intéressent cet aspect central du brevet : celles détaillant l'obligation d'enseigner pesant sur le maître et celles qui, de manière complémentaire, mettent à charge de l'apprenti une obligation d'apprendre.

L'obligation d'enseigner pèse en principe personnellement sur le maître, même s'il n'est pas titulaire d'une maîtrise ${ }^{151}$. Quelques rares brevets précisent en effet que le « maître » ne l'est pas, ou plutôt qu'il n'est pas titulaire d'une maitrise, mais que l'apprenti et ses représentants le savent et que cela n'a pas d'incidence sur le bon déroulement de l'apprentissage. C'est ainsi que Pierre Lafont, maréchal ferrant à Vinça, prend Jean-Pierre Macari en apprentissage alors qu'il ne dispose pas de maîtrise. En l'occurrence, le père de l'apprenti se porte garant de l'accomplissement des obligations de son fils en connaissance de cause puisque par deux fois le notaire précise qu'il s'engage "quand bien même il soit de sa connoissance que ledit Lafont comparant n'est pas reçu maître maréchal » et plus loin dans le contrat, "quand bien il sçache positivement que ledit Lafont ne soit pas reçu maître ${ }^{152}$. Cet apprenti n'a donc pas de véritable maître pour lui dispenser son savoir alors que d'autres peuvent bénéficier des lumières de deux maitres travaillant dans le même atelier. Il s'agit là d'un cas unique parmi les brevets envisagés où Denis Laporte et Pierre Darnis, cordonniers, travaillant ensemble à Opoul, acceptent d'enseigner ensemble leur métier à Jacques Chavanete ${ }^{153}$.

Par exception cette obligation peut peser sur une autre personne, notamment un membre de la famille du maitre. Les ateliers familiaux sont sans doute bien plus nombreux que ne le laissent penser les brevets. La participation d'un fils travaillant au sein de l'atelier ou boutique n'est très certainement pas systématiquement consignée par écrit. Cas rare, Emmanuel Marsal, maître tourneur de chaises, promet d'enseigner " ou faire enseigner par François Marsal son fils ", présent à l'acte et qui

151 - II doit en principe être maitre, avoir ouvert boutique et ne pas avoir atteint le nombre maximal d'apprentis prescrit par les statuts. B. GaLLINATO, op. cit., p. 77.

152 - 3E 19/791, f 378, n 154, brevet du 31 août 1788 passé devant Me Martin Molins, notaire à Vinça.

153 - 3E 15/80, f० 149, brevet du 19 avril 1779. 


\section{LA SUBORDINATION DE L'APPRENTI À TRAVERS LES BREVETS...}

s'engage également, le métier de tourneur de chaises ${ }^{154}$. Cas plus rare encore, une femme peut participer à la formation d'un apprenti. Ainsi Thérèse Catala s'engage avec son fils, Jean Bes et Catala, à enseigner le métier de tanneur, autrefois exercé par leur défunt mari et père respectif, à Modeste Sicart ${ }^{155}$.

L'intervention d'un tiers dans la formation de l'apprenti est plus fréquente en Cerdagne où sept brevets signalent qu' " un ouvrier en état " ${ }^{156}$ ou " ouvrier au fait ${ }^{157}$ peut suppléer au maître dans cette tâche.

En effet, le maître se doit normalement d'accueillir l'apprenti en sa maison et boutique et, selon la formule employée par Me Jean Felip-Barrère, notaire à Prades, le traiter " en bon père de famille " ${ }^{158}$, voire en "bon maître et père de famille ${ }^{159}$, pour lui enseigner son métier avec " douceur ${ }^{160}$. Ce notaire combine même ces expressions à deux reprises, écrivant dans la clause la plus développée que le maître va " enseigner son métier avec les attentions et la douceur d'un bon père de famille ${ }^{161}$. Ces formules sont sans doute des rémanences de pratiques plus anciennes, d'une époque où le maître se substituait au père de l'apprenti en devenant à la fois un éducateur moral et professionnel, un temps où l'apprenti était " loco filii à l'égard de son maître " ${ }^{162}$. Ces formules familiales sont d'ailleurs immédiatement suivies du rôle pédagogique incombant au maître. Il doit enseigner son métier " autant ", " de la meilleure manière " ou " du mieux " " qu'il luy sera possible ». Il ne peut en effet transmettre que ce qu'il sait lui-même, professant " suivant ses lumières " ${ }^{163}$ et " en conscience " ${ }^{164}$. Afin de garantir la meilleure formation possible, les statuts des corps limitent généralement le nombre des apprentis que peut accueillir concomitamment un maître ${ }^{165}$. En Roussillon, généralement il n'y a qu'un apprenti par boutique mais il peut arriver qu'exceptionnellement il y en ait deux ${ }^{166}$. Le maître peut ainsi entièrement se consacrer

$\mathbf{1 5 4}-3 \mathrm{E} 13 / 21, \mathrm{n}^{\circ} 135$, brevet du 16 mai 1781

155 - 3E 23/46, f० 129, brevet du 5 octobre 1785 passé devant Me Dominique Bordes, notaire à Prades.

156 - Ce sont les brevets des 14 février 1783 (3E 56/160, n 58), 6 novembre 1784 (3E 56/60, n 314), 24 mai 1789 (3E $56 / 61, n^{\circ} 535$ ) et du ler juin 1789 (3E $56 / 61 n^{\circ} 537$ et 538).

$157-3 E 56 / 160, n^{\circ} 120$ et $3 E 56 / 161, n^{\circ} 88$, brevets des 24 avril 1783 et 2 mai 1784.

158 - Cette formule est employée, outre Me Jean Felip-Barrère qui l'utilise dans sept brevets des onze quíl rédige pour cette période, par Me Sauveur Jaume notaire de Perpignan, qui ne l'emploie que dans deux de ses vingt-neuf brevets recensés pour cette période. Elle est également usitée à Toulouse à la même époque où se rencontre également la formule selon laquelle le maître doit enseigner à l'apprenti « comme si c'était son propre fils », voir J. DE BONNE, op. cit., p. 50.

159 - Me Jean Felip-Barrère utilise cette formulation deux fois.

160 - 3E 21/463, nं144, f 304, brevet du 16 octobre 1779.

$161-3 E 21 / 467, n^{\circ} 114, f^{\circ} 209$, brevet du 22 avril 1783.

162 - J. DASSONVILLE, op. cit. p. 64.

163 - Deux brevets du même notaire, Me Jacques Bonnaventure Xinxet notaire à Collioure, utilisent cette formulation : celui du 24 août 1785 (3E 30/103) et celui du 20 février 1787 (3E 30/105).

164 - Formule employée par deux fois par Me Joseph Nicolau-Coromina, notaire à Villefranche-de-Conflent (3E 24/54, $n^{\circ} 38$ et 40 , brevets des 3 et 8 juin 1788).

165 - Cela doit également garantir un bon traitement de l'enfant et éviter l'encombrement de la profession. E. MARTIN SAINT-LÉON, op. cit., p. 80. C'est le cas par exemple en Poitou, voir P. BOISSONNADE, op. cit., p. 39.

166 - Une fois que le premier apprenti approche du terme de sa période de formation, un second est parfois accueilli par le maître « pour dégrossir le nouvel élève, avant le départ de l'ancien ». J. DASSONVILLE, Le contrat d'apprentissage et son régime légal, th., dr. Lille, 1904, p. 24 ; P. BoISSONNADE, op. cit., p. 41. Plusieurs maîtres roussillonnais agissent de la sorte, 
à son seul élève. Il arrive encore plus exceptionnellement qu'un maître prenne davantage d'apprentis ${ }^{167}$ : c'est sans doute le cas dans les fabriques de Cerdagne espagnole ${ }^{168}$ mais pas seulement. Ainsi, du 18 février au 21 mai 1782, Antoine Boyer, qui tient une fabrique dans le village de Thuir appartenant au chevalier Jean Coll de Vivès, engage successivement quatre apprentis tourneurs mouleurs faïenciers puis un apprenti peintre faïencier. Il ne les loge pas et ne les nourrit pas non plus. Il leur verse successivement à chacun quatre, six puis huit sols par jour de formation au cours de leurs trois années de formation. On est bien loin d'une ambiance familiale mais plutôt dans un atelier rural de type préindustriel ${ }^{169}$.

L'apprenti se doit, de son côté, de recevoir " avec docilité les leçons ${ }^{170}$ de son maître. L'enseignement est donc inhérent aux capacités et à la bonne volonté de l'apprenti. Les brevets précisent que l'enseignement doit être dispensé « autant que [l'apprenti] aura de(s) dispositions pour apprendre ${ }^{171}$ ou encore " de la meilleure manière que l'apprenti pourra apprendre $»^{172}$, voire que l'apprenti " voudra apprendre $»^{173}$ ou autant qu'il aura « la capacité et docilité suffisantes ${ }^{174}$. Ces formules renvoient autant aux capacités de l'apprenti qu'à son obligation d'obéissance ${ }^{175}$.

Tout d'abord, pour avoir le droit d'entrer dans certaines formations des prérequis sont parfois nécessaires. Ainsi est-il demandé aux apprentis imprimeurs de fournir un certificat de maitrise du latin et du grec que délivre le recteur de l'université ${ }^{176}$. Pour d'autres formations, sans doute est-il demandé de maitriser l'écriture et la lecture. Voilà pourquoi un maître chirurgien prenant un apprenti doit " le faire appliquer a bien lire et ecrire " ${ }^{177}$. De la même manière, un maitre graveur ciseleur ${ }^{178}$ ou orfevvre ${ }^{179}$ peut commencer par apprendre à son apprenti le dessin ou bien lui laisser le temps nécessaire tous les jours pour qu'il l'apprenne. L'apprenti continue donc parfois à aller à l'école en même temps qu'il commence à apprendre un métier ${ }^{180}$.

mais l'exemple le plus illustratif est celui de Jean Sallent boulanger de Prades, qui systématise ce procédé. II contracte en effet quatre brevets de deux ans durant cette période, prenant un nouvel apprenti dès lors qu'il reste entre un et six mois de formation à l'ancien. Les brevets sont datés des 18 février 1783, ler janvier 1785, 9 juillet 1786 et 18 mai 1788 (3E $23 / 45, f^{\circ} 37,3 E 23 / 46, f^{\circ} 1,3 E 23 / 47, f^{\circ} 87$ et $3 E 23 / 48, n^{\circ} 39, f^{\circ} 551$.

167 - Pour le Poitou, voir P. BoISSONNADE, op. cit., p. 40-41.

168 - George Carbonnel, fabricant de bas de coton dans l'enclave espagnole de Livia, prend trois apprentis en 1782,

1783 et 1784 pour les former durant trois à quatre ans chacun. En 1784, trois apprentis lau moins et à moins d'une résiliation anticipée de l'un des premiers brevets) travaillent donc pour lui.

169 - Brevets du 18 février, 10 mars, 13 mars, 16 mars et 21 mai 1782 (3E 26/97, n 50, 80, 86, 87 et 192). Ces ateliers ruraux échappent à la réglementation corporative partout en France. J. DASSONVILLE, op. cit., p. 25.

170 - 3E $21 / 463, n^{\circ} 110, f^{\circ} 230$, brevet du 25 juillet 1779.

$171-3 E 13 / 21, n^{\circ} 135$, brevet du 16 mai 1781.

172 - 3E 24/54, nं38, brevet du 3 juin 1788

173 - 3E 19/736, f 493, nं 230, brevet du 13 novembre 1785.

174 - 3E 14/59, nं 534, brevet du 22 août 1780.

175 - Voir infra II B.

176 - 3 E 15/89, f 333 et 3 E 15/98, f 297, brevets du 19 août 1783 et du 28 octobre 1788

$177-3 E 21 / 467, n^{\circ} 161, f^{\circ} 317$, brevet du 4 juillet 1783.

178 - 3 E 25/5, nं 146 et $3 E 25 / 8, n^{\circ} 114$, brevets des 15 septembre 1785 et 28 août 1788 .

179 - 3E 13/19, nं 301, brevet du 16 septembre 1779.

180 - 3E 26/24l, f 230 , brevet du 10 octobre 1788. 
En dehors de ces quelques situations particulières, les conditions dans lesquelles doit se dérouler l'enseignement ne sont que rarement détaillées. Tout semble relever de la pratique. Le maître doit enseigner son métier " ainsi qu'il est d'usage et de coutume ${ }^{181}$ ou " suivant l'usage du païs ${ }^{182}$. Cette dernière formulation, sibylline et laconique, vise à éviter toute omission dans la formation que doit dispenser un maître pareur. Il s'engage en effet à " enseigner le métier de pareur soit en ce qui concerne la preparation des laines soit pour tisser les draps pour leur donner une couleur pour les arranger et preparer et enfin pour tout ce qui concerne le métier de pareur suivant l'usage du païs ${ }^{183}$. Concrètement, le maître utilise une technique démonstrative. L'apprenti doit observer la façon dont procède le maître. Le maitre doit donc " montrer et enseigner " ${ }^{184}$ ou bien " enseigner et montrer ${ }^{185}$ son métier. S'il ne le fait pas, l'apprenti est autorisé à quitter la boutique ${ }^{186}$.

Quatre brevets seulement évoquent la fourniture par le maitre des outils nécessaires à l’apprentissage du métier ${ }^{187}$. Ainsi le maître doit " fournir [...] tous les outils pour et nécessaires pour apprendre le maitier ${ }^{188}$. Il est même parfois prévu que l'apprenti puisse se servir des outils de son maitre pour réaliser des travaux sur ordre de son père ${ }^{189}$. Le quatrième brevet évoquant ce point le fait après avoir précisé que tout ce qui concerne la nourriture et l'entretien de l'apprenti sera assumé par son père et que seule la fourniture des outils relèvera du maitre. Ce brevet énonce ainsi "que ledit Lafont père s'oblige de fournir audit son fils pendant ledit tems tous les aliments et entretiens, ledit Sr Gayme ne devant entrer pour rien dans ces objets si ce n'est a la fourniture des outils nécessaires ledit métier ${ }^{190}$. Mais aucun brevet ne prévoit de dotation de l'apprenti en outils à la fin de son apprentissage par son maître afin de faciliter son entrée dans le monde du travail ${ }^{191}$, ni de fourniture des outils par les parents de l'apprenti ${ }^{192}$ ou son représentant au début de la formation $^{193}$.

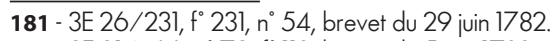

182 - $3 E$ 21/466, n 79, f $f^{\circ} 112$, brevet du 5 juin 1782.

183 - Ibid.

184 - 3E 12/96, nं 202, brevet du 8 novembre 1785. Cinq autres brevets reprennent exactement cette formule.

185 - 3E 21/435, n 29, f 59, brevet du 14 février 1780. Cette formule, inversée par rapport à la précédente, est la seule du genre.

186 - En Poitou cela est formulé par des statuts de métiers et contrats d'apprentissage, voir P. BoISSONNADE, op. cit., p. 51, n. 4.

187 - Pour le Poitou, voir P. BoIsSONNADE, op. cit., p. 51.

188 - $3 E$ 40/661, f 43 , brevet du 13 février 1780. Le même notaire, Me Louis Companyo de Céret, emploie une formule assez proche dans le brevet du 29 août 1780 (3E 40/661, f ${ }^{\circ} 268$ ).

189 - 3E 44/65, n¹92, brevet du 2 novembre 1784.

190 - 3E 25/6, n¹42, brevet du 20 juillet 1786.

191 - Cela se pratique en Bourgogne ou en Orléanais à la fin du Moyen âge. Voir P. DiDIER, op. cit., p. 44-45 et F. MiCHAUD-FREJAVILL, « Bons et loyaux services... op. cit., p. 206.

192 - Joseph De Bonne cite un exemple contraire de contrat d'apprentissage de faiseur de petits souliers du 14 octobre 1698. J. De BONNE, op. cit., p. 30.

193 - Alain Degage relève deux brevets seulement, parmi les cinq cent huit qu'il a recensés à Sète au XVIIle siècle, prévoyant un tel cas de figure. A. DEGAGE, op. cit., p. 28. 
Les quelques précisions qu'offrent les brevets révèlent que le maitre doit se montrer assidu dans cette mission. Jacques Rufiandis, chirurgien de Mosset, doit ainsi enseigner son métier " avec toute assiduité et sans interruption " à Jean Blanch pendant quatre années ${ }^{194}$.

L'étendue de l'enseignement à dispenser tient au métier auquel se destine l'apprenti. Le maître apprend au jeune garçon placé dans sa boutique " tout ce qui concerne sa profession ${ }^{195}$ comme " le commerce ${ }^{196}$ de menuisier ou "l'état de chirurgien ${ }^{197}$. Il doit enseigner le métier " en tous ses chefs ${ }^{198}$ et, selon une formule très usitée, "sans lui en rien cacher ", voire " sans lui en rien cacher ni celer ${ }^{199}$.

Ainsi l'apprenti doit être à la fin de la période d'apprentissage capable d'exercer la profession que le maitre vient de lui enseigner. Un certificat attestant du bon déroulement de la formation peut in fine être délivré par le maître ${ }^{200}$. Deux brevets prévoient que le maître doit enseigner son métier et « veiller soigneusement et avec attention à ce qu'il [l'apprenti] soit capable et en état à la fin du présent apprentissage de mériter d'être admis et reçu maître ${ }^{201}$. Il s'agit de deux brevets passés le même jour, le 27 février 1789 et par le même notaire, Me Jean Mundi-Albafulla notaire de Perpignan, par lesquels deux marchands orfèvres de Perpignan prennent pour apprentis leurs propres fils. Un brevet prévoit tout simplement que le jeune homme a été placé « en apprentissage de Maître charron " ${ }^{202}$. Malgré la rareté de ce type de clause, elle n'est pas inutile en ce qu'un apprenti cantonné à des tâches subalternes, pourrait se trouver au terme de l'apprentissage inapte à exercer le métier qu'il est censé avoir appris ${ }^{203}$. A l'inverse un apprenti peut être déjà au fait du métier qu’il est supposé apprendre. Un acte de résiliation du 3 octobre 1788 énonce ainsi que l'apprenti nommé Galderich Fabre, placé le 20 septembre 1788 chez Dominique Manent maitre tailleur de Vinça ${ }^{204}$, soit deux semaines auparavant, a dit à son maittre " qu'il ne vouloit plus continuer son apprentissage en sa maison pour se trouver déjà assés instruit pour le temps qưil avoit travaillé chés ledit Paul Fabre son père ", également tailleur, habitant Marquixanes ${ }^{205}$.

\footnotetext{
$194-3 E 21 / 467, n^{\circ} 161, f^{\circ} 317$, brevet du 4 juillet 1783 .

195 - Formule employée par Me Dominique Bordes, notaire de Prades.

$196-3 E 19 / 784, f^{\circ} 18, n^{\circ} 8$, brevet du 3 janvier 1781.

$197-3 \mathrm{E} 36 / 113, \mathrm{n}^{\circ} 140$, brevet du 16 novembre 1787

198 - Une formule employée à deux reprises par Me Alexis Conte, notaire de Perpignan, dans les brevets des 16 août 1779 et 30 mars 1780 (3E 5/75, n² $\left.293 ; 3 E 5 / 76, n^{\circ} 165\right)$.

199 - C'est une règle générale. J. DASSONVILE, op. cit., p. 55.

200 - Notamment pour les enfants placés en apprentissage par la Miséricorde. Voir C. JuHEL, op. cit., p. 131-132. Cela se pratique aussi à Toulouse à cette époque. J. De BONNE, op. cit., p. 53.

$201-3 E$ 13/51, 2e registre, nos 78 et 79.

202 - 3 E 10/102, f $444 v^{\circ}$, brevet du 14 novembre 1779.

203 - Louis Morin donne l'exemple d'un jeune homme qui contracte un brevet d'apprentissage de chapelier d'une durée d'un an et demi après avoir subi un premier apprentissage de quatre ans. L. MoRIN, op. cit., p. 9-10.

204 - 3E 19/791, nं 164, f 404.

205 - 3E 19/791, nं 169, f० 417.
} 
Cet enseignement exhaustif garantit une formation complète et limite par la même occasion l'étendue de l'autorité du maitre sur son apprenti puisqu'il ne peut lui donner d'ordres qu’à propos du métier qu'il enseigne et ne doit pas le divertir à d'autres tâches ${ }^{206}$. La spécialisation de la formation apparaît essentielle aux yeux des rédacteurs de ces brevets ${ }^{207}$. Il est donc très rare qu'un maître prévoie de ne pas enseigner une partie de son savoir. Seuls trois brevets mentionnent une telle restriction ${ }^{208}$. Le premier concerne Joseph Girau maître bourrelier de Perpignan qui prend François Patruix en apprentissage le 9 septembre 1782 en promettant de lui enseigner « tout ce qui est dépendant dudit métier sans lui rien cacher à l'exception du travail des harnais des chevaux ou mules de charrette que ledit Girau ne lui enseignera point ${ }^{209}$. Aucune explication n'est fournie par le brevet sur ce point. Les deux autres brevets relèvent d'une forme de spécialisation que se réserve le maître, en l'occurrence Joseph Julia, maitre perruquier de Perpignan. En effet, le 26 octobre 1781 Jean-Baptiste Angles, alguazil au Conseil souverain ${ }^{210}$, place son fils prénommé Ange en apprentissage chez ce dernier qui " promet enseigner ledit métier sans lui en rien y sceller à l'exception néanmoins des accomodages de femmes que ledit Julia se réserve par exprès " ${ }^{211}$. De la même manière, le 4 juin 1783, il s’engage à enseigner son métier à Gaspard Boudiere «à l'exception des accomodages des femmes à quoi ledit $\mathrm{Sr}$ Julia ne s'engage pas ${ }^{212}$.

Il est vrai que certains métiers distinguent les clientèles d'hommes et de femmes : il existe des tailleurs d'habits pour femmes et des cordonniers pour femmes. En conséquence certains brevets d'apprentissage précisent que le maître doit enseigner son métier uniquement "pour femmes ». Huit brevets présentent cette caractéristique : six pour des cordonniers et deux pour des tailleurs. La plupart du temps le maître est lui-même spécialisé. Ainsi " Joseph Tore maître cordonnier pour femmes " s'engage le 17 juin 1781 à former Joseph Carrère à ce métier-là ${ }^{213}$ et le 5 octobre 1789 à former Augustin Montagne ${ }^{214}$. Tout comme Joseph Prunet, maître cordonnier pour femme, prend le 14 avril 1784 François

\footnotetext{
206 - Voir infra ll B.

207 - II semble qu'ailleurs, en Bourgogne aux XIVe et XVe siècles, ce soit la qualité de la formation qui prime sur la spécialisation. Un tapissier peut enseigner la teinturerie. Un même artisan peut être drapier, épicier et chaussetier, et enseigner ces trois métiers, ce qui se justifie dans « une économie de pénurie où la spécialisation du travail est encore embryonnaire ». P. DIDIER, op. cit., p. 48.

208 - Ces restrictions, bien que rares, n'ont rien d'original. Il en existe dans toutes les professions partout en France. Voir L. MORIN, op. cit., p. 12.

$209-3 \mathrm{E} 13 / 22, \mathrm{f}^{\circ} 403, \mathrm{n}^{\circ} 263$.

210 - Les alguazils sont des huissiers officiant au Conseil souverain de Roussillon. Voir F.P. BLANC, « Les alguazils en la Cour du Conseil souverain de Roussillon : une institution atypique sous l'Ancien Régime (1660-1790) », in G. LARGUIER (dir.), Métiers... op. cit., p. 219-232.

$\mathbf{2 1 1}$ - 3E $5 / 78, n^{\circ} 355$

212 - 3E 5/80, nं 258.

$213-3 E 5 / 78, n^{\circ} 210$

214 - 3E $14 / 77$ n 734.
} 
Tallada en apprentissage pour lui enseigner cette même activité ${ }^{215}$. Dans d'autres cas il semble que le maître ne soit pas spécialisé mais dispense un enseignement spécialisé. Ainsi le 22 mai 1785 Claude Territs est placé « en apprentissage de tailleur d'habits pour femmes " par sa mère chez Louis Cayrou maître tailleur de Perpignan ${ }^{216}$ alors que le second brevet d'apprentissage de tailleur pour femme est conclu avec "François Mori maître tailleur pour femme » ${ }^{217}$. De même, Charles Lefevre maître cordonnier de Perpignan s'engage à enseigner le métier de cordonnier pour femme à Antoine Bernard le 5 août $1779^{218}$. Par exception, un brevet, signé le 9 mai 1779 en faveur de Dominique Saularé et Glory, précise que Jacques Gasch maître cordonnier va lui apprendre " le métier de cordonnier tant pour homme que pour femme $»^{219}$.

\section{B. L'obligation de travailler pour le maître}

Sous l'Ancien régime, travailler pour un maître implique non seulement de lui obéir $^{220}$ mais encore de ne servir que ses intérêts.

Depuis le Moyen âge, il ne fait aucun doute que l'apprenti doit «faire gré à son maître $»^{221}$. Il doit être obéissant, docile ${ }^{222}$ et fidèle. Dans les brevets cette obligation d'obéissance est formulée de manière très stéréotypée. L'immense majorité des contrats précise que le jeune garçon s'engage à "obéir audit son maître en touttes choses licites et honnêtes concernant ledit métier " ${ }^{223}$. Le maître doit être un modèle pour lui. A sept reprises seulement le brevet mentionne que l'apprenti est « tenu d'etre soumis docile et obéissant audit [...] son maitre tant qu'il sera en apprentissage et sur tout ce qu'il luy commandera de licite et honnette ${ }^{224}$ tandis que dix autres brevets, tel que celui rédigé par Me Serra le 26 mai 1785, affirment que l'apprenti est tenu « de faire tout ce que pareils apprentis sont obligés de remplir concernant ledit métier ${ }^{225}$. L'apprenti doit "servir audit métier avec loyauté et fidélité » ${ }^{226}$, obtenant même en fin d'apprentissage un " certificat de loyauté et fidélité " attestant qu'il a bien accompli son temps d'apprentissage en faisant

$215-3 E$ 5/81, n 123.

$216-3 E 25 / 5, n^{\circ} 74$.

217 - 3E 14/66, n 181, brevet du 29 mars 1784. Assez curieusement ce brevet laisse entendre qu'il n'y a pas de maître tailleur pour femme à Perpignan pour justifier ce choix professionnel mais qualifie pourtant ainsi François Mori.

$218-3 E 13 / 19, n^{\circ} 260$.

219 - 3E 5/75, nं204.

220 - Selon Philippe Didier, en Bourgogne à la fin du Moyen âge, « tous les devoirs de l'apprenti [...] semblent se ramener à une obligation d'obéissance ». P. DIDIER, op. cit., p. 39

221 - J. DASSONVILLE, op. cit., p. 51.

222 - Le terme est employé à Sète à cette époque. A. DeGAGE, op. cit., p. 28

223 - Cette clause se pratique à Toulouse aussi où l'on recourt également à des clauses faisant référence « aux bonnes mœurs », voire à « la crainte de Dieu ». J. De BONNE, op. cit., p. 51.

$224-3 E 32 / 3, f^{\circ} 361$, brevet du 4 novembre 1785. C'est la formulation retenue par Me François Maria, notaire à Thuir, dans les quatre brevets qu'il rédige au cours de cette période.

225 - 3E 14/68, nं 366.

$226-3 E$ 15/80, f 226, brevet du 25 mai 1779. 
montre de ces qualités ${ }^{227}$. Cette obligation d'obéir contractée par l'apprenti vise le maître mais aussi les ouvriers travaillant avec lui ${ }^{228}$. Ainsi, dans son brevet d'apprentissage de fabricant de bas, Pierre Calvet accepte "d'obéhir [à] son maître et de travailler assiduement aux ordres de son dit maître et à ceux d'un ouvrier au fait que ledit Bonnaventure Calvet s'oblige à tenir chez luy pour apprendre audit apprenti ledit métier ${ }^{229}$. Du point de vue du maître, cette obligation implique qu'il donne des ordres à l'apprenti pour le former "sans le divertir à d'autres ouvrages que ceux relatifs audit métier $»^{230}$. Enfin, pour se montrer fidèle envers son maître, l'apprenti doit " éviter son dommage ", voire "l'en avertir s'il vient à sa connaissance ${ }^{231}$. Il doit veiller à ce que son maître ne subisse aucun dommage matériel. Rien ne doit lui être volé dans son atelier ou boutique. Les parents ou responsables de l'apprenti s'en portent garants ${ }^{232}$.

En outre, bien qu'il s'agisse d'un élément central de ce contrat de formation professionnelle, l'obligation de travailler n'apparaît que rarement de manière littérale dans les brevets. Elle est rattachée à l'engagement que souscrit l'apprenti de prendre " les intérêts de son maître à cœur comme propres " et de "faire son profit ». Il lui est donc interdit de lui causer du tort ${ }^{233}$ ou de travailler dans une

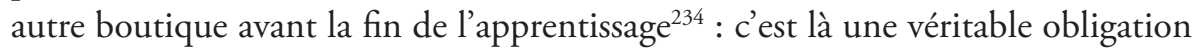
de non concurrence pesant sur lui en raison de la conclusion du brevet ${ }^{235}$. Les formules sont assez semblables : l'apprenti ne peut " aller ailleurs pendant ledit temps servir d'autres personnes ${ }^{236}$ ou $"$ toute autre personne $»^{237}$.

L'étendue matérielle de cette interdiction est rarement précisée. Les activités proscrites ne sont pas évoquées par les brevets en dehors de celui souscrit par Laurent Just, apprenti placé chez Laurent Forga tisserand à lin de Palalda, qui mentionne qu'il ne pourra aller travailler pendant son temps d'apprentissage

$\overline{227}$ - Vingt-deux brevets prévoient la délivrance d'un « certificat d'assiduité et de loyauté » par le maitre en fin de formation. Ils concernent tous des enfants placés par l'hôpital de la Miséricorde. Voir C. JUHEL, op. cit., p. 131-132.

228 - B. Gallinato, op. cit., p. 83.

229 - 3E 56/160, nं 120 , brevet du 24 avril 1783.

230 - Cette formule n'intervient qu'à trois reprises dans les brevets. Une première fois sous la plume de Me Jean Felip-Barrère, notaire à Prades (3E 21/467, $n^{\circ}$ 114, $f^{\circ}$ 209, brevet du 22 avril 1783), puis deux fois parmi les nombreux brevets rédigés par Me Sauveur Jaume à Perpignan (brevet du 25 mai 1779, 3E 15/80, f 226 ; brevet du 12 janvier 1786, 3E 15/94, f 19). Pour Sète, voir A. DegaGe, op. cit., p. 28.

231 - Dix brevets seulement comportent cette dernière précision qui se retrouve dans les contrats toulousains de l'époque. J. DE BONNE, op. cit., p. 52.

232 - J. DE BONNE, op. cit., p. 47-48.

233 - Aucun contrat n'évoque les dégâts que pourrait causer un apprenti chez son maître. C'est pourtant le cas à Troyes au XVIle siècle. L. Morin, op. cit., p. 17-18.

234 - II n'y a aucun exemple d'interdiction de travailler ailleurs que dans la boutique du maitre une fois le terme du contrat échu. Philippe Didier en signale en Bourgogne à la fin du Moyen âge, un apprenti se voyant interdire de travailler en Bourgogne durant les cinq années suivant la fin de sa formation. P. DIDIER, op. cit., p.41.

235 - En Poitou ou à Bordeaux, le maître embauchant un apprenti en fuite s'expose à des peines d'amende (B. GALLINATO, op. cit., p. 98.), d'interdiction temporaire, voire au paiement de dommages-intérêts au maître floué (P. BOISSONNADE, op. cit., p. 49-50).

236 - 3E 44/60, nं 137, brevet du 2 aoôt 1779.

237 - Voir par exemple le brevet du ler août 1784, 3E 44/65, n 133. 
" servir [un] autre ticeran $»^{238}$, ce qui a contrario laisse penser qu'il pourrait exercer d'autres arts ou métiers.

L'étendue géographique de l'interdiction correspond à la province roussillonnaise. Cette délimitation ressort de l'ensemble des dispositions des brevets consultés et notamment de l'interdiction de travailler dans une autre boutique de la province ou encore de l'absence de responsabilité des parents lorsque l'apprenti quitte la province ${ }^{239}$.

L'étendue temporelle de l'interdiction n'est pas non plus détaillée car il semble que, tout naturellement, cette obligation dure aussi longtemps que l'apprentissage lui-même. Ainsi, si l'apprenti s'absente de chez son maître et que le brevet ne prévoit rien pour son remplacement ou pour dédommager le maître, il ne pourra plus travailler dans la province. Un temps d'apprentissage inachevé peut donc produire des effets très longtemps après la signature du brevet. C'est ce que semble établir le brevet du 11 novembre 1779 plaçant François Ville chez Antoine Biu maître barbier perruquier baigneur et étuviste de Perpignan, précisant " que si pendant ledit apprentissage le même Ville venait à s'absenter de chez ledit Biu ou quitter cette ville sans une permission expresse de ce dernier et sans que celui-ci y eut donné lieu, en quel temps que ledit Ville revienne en cette ville ou quand bien même il ne partirait pas d'icelle, il ne pourra en aucune façon entrer dans aucune autre boutique qu'il n'ait complété chez ledit Biu lesdites deux années ${ }^{240}$. Un acte de résiliation confirme que cette obligation de non concurrence perdure aussi longtemps que la durée fixée par le brevet n'a pas été totalement accomplie. En effet, Joseph Glori de Peyrestortes a été placé en apprentissage pour deux ans chez Marc Bonet marchand tanneur de Perpignan le 5 juin $1786^{241}$. Ce brevet est résilié par un acte du $1^{\text {er }}$ octobre 1786 aux termes duquel le maître ne s'y « prette qu'à la condition que ledit Joseph Glori apprenti ne pourra point travailler de taneur dans cette province de Roussillon de façon que s'il hasardoit de ce faire ledit Bonet rentreroit dans le droit de demander l'exécution dudit brevet non obstant le présent acte ${ }^{242}$. La résiliation du brevet est donc soumise à une condition résolutoire, le respect de l'obligation de non concurrence.

Pour Alphonse Drapé cette obligation, de non concurrence est une règle absolue ${ }^{243}$ mais il existe une toute petite exception, celle du " chômage technique ", lorsque le maître est dans l'incapacité de donner du travail à réaliser à son apprenti. Ce cas de figure est envisagé uniquement par certains maîtres de l'enclave de Livia. Tous les maîtres ne souhaitent en effet pas libérer leurs apprentis en de

238 - 3E 44/70, n 148, brevet du 24 mai 1789.

239 - Voir supra.

240 - 3E 5/75, nं 405.

$241-3 E 14 / 70, n^{\circ} 396$.

242 - 3E 14/71, n 626.

243 - A. Drapé, op. cit., p. 122. 
pareilles circonstances. A titre d'exemple, le brevet du 2 mai 1784 dispose qu'au cas où le maitre " restat une semaine sans donner de l'ouvrage audit apprenti, ce dernier ne pourra point pour raison de ce quitter ledit son maitre, mais bien exhiger cette semaine là ainsy qu'il aurait gagné la semaine précédente $»^{244}$. De même un brevet du 14 février 1783 énonce qu' " au cas ledit apprenti fut obligé à suspendre quelques jours son travail, faute par ledit Calvet de manquer des matheriaux pour faire travailler, ledit Calvet sera tenû à payer également audit Visas [son apprenti] sa journée ordinaire, restant ledit Visas chez ledit Calvet et non ailleurs les jours qu'il ne pourroit point travailler ». Seul un brevet, rédigé le 14 septembre 1783, mentionne in fine la " faculté aussy audit apprenti de s'en aller dans une autre fabrique au cas son maitre ne luy tiendroit point ouvrage à luy donner tout de suitte $»^{245}$. En ce dernier cas le maître préfère économiser les demi-salaires des journées chômées plutôt que de conserver à disposition cette main d'œuvre bon marché. Il est le seul à faire ce choix.

La règle est donc la même à Livia que dans la province de Roussillon. Les brevets d'apprentissage rédigés par les notaires de Saillagouse précisent que si l'apprenti placé à Livia quitte son maître pour aller travailler dans une autre fabrique que celle de son maitre, lui et la personne l'ayant placé seront tenus de payer 14 sols par jour " pendant tout le temps ouvrier que [l'apprenti] aura soustrait dudit apprentissage $»^{246}$ ou bien " cinq sols monnoye barcelonnaise courante en Espagne par jour qu'il manquera à compléter le terme dudit apprentissage $»^{247}$, soit le salaire d'un compagnon.

En Roussillon ${ }^{248}$ les brevets prévoient généralement qu’en pareil cas les parents de l'apprenti «s'oblige[nt] à maintenir, nourrir et entretenir un garçon payer au lieu et place de leur fils pour achever de compléter audit Calvet [son maître] le temps de l'apprentissage dudit [leur fils] ou bien luy refaire tous dépends dommages et intérêts ${ }^{249}$. De la même manière, Dominique Calvet, voiturier, s'engage " à faire parachever le temps de l'apprentissage par un autre ouvrier au lieu

244 - 3E 56/161, nं 88.

$245-3 E 56 / 160, n^{\circ} 278$.

246 - 3E 56/60, n' 314, brevet du 6 novembre 1784. Cette même formule est reprise dans les trois autres brevets rédigés par François Nicolau, notaire à Saillagouse : voir le brevet du 24 mai 1789 (3E 56/61, n 535) et les deux brevets du ler juin 1789 (3E 56/61 nos 537 et 538). On la retrouve aussi dans certains brevets rédigés par Joseph Galard, autre notaire de Saillagouse : brevets du 8 mai 1782 (3E 56/159, n 114) et du 14 septembre 1783 (3E 56/160, n²78).

247 - 3E 56/159, n² 248, brevet rédigé par Joseph Galard le 29 septembre 1782. Une formule reprise par Galard dans deux autres brevets pour toute absence de l'apprenti (3E 56/16l, n' 88 et 3E 56/165, brevets du 2 mai 1784 et 25 février 1788).

248 - A titre d'exemple, le brevet rédigé le 15 octobre 1781 prévoit « si pendant le cours dudit apprentissage ledit Joseph Razongles venoit à quitter la boutique dudit Blanich pour aller travailler ailleurs en cette province, ledit Blanich pourra prendre un garçon à sa place aux fraix et dépens dudit Razongles père » (3E 22/253, $\mathrm{n}^{\circ} 231, f^{\circ} 271$ ). II faut souligner qu'à la différence de ce qui se pratique à Livia l'interdiction de travailler ailleurs n'est pas limitée à la province de Roussillon et s'applique à l'Espagne, notamment à Puycerda.

249 - 3E 56/160, n 57, brevet rédigé par Joseph Galard le 14 février 1783. Formule reprise presqu'à l'identique dans un autre brevet qu'il rédige le même jour (3E 56/160, n 58). 
et place de son fils en luy payant la moitié du travail $»^{250}$. Cette moitié de salaire permet de compléter le salaire habituellement versé par le maître à l'apprenti et qui correspond dans les fabriques de Cerdagne à la moitié du salaire d'un compagnon. Le compagnon remplaçant l'apprenti doit ainsi percevoir deux moitiés de salaire, l'une du maitre et l'autre de la famille de l'apprenti, soit un salaire complet. Il s'agit là d'une pratique répandue. Les parents de l'apprenti doivent tenter de le faire revenir dans la boutique et en cas d'échec, le maitre peut prendre un ouvrier ou compagnon à sa place à charge par eux de le rémunérer. Il importe seulement de ne pas se précipiter. Si un apprenti s'absente sans raison légitime durablement de la boutique, le maître doit attendre huit ${ }^{251}$ ou quinze ${ }^{252}$ jours avant de le remplacer ${ }^{253}$.

En dehors des cas de pénurie de travail, les hypothèses dans lesquelles un apprenti peut aller travailler pour un autre maître sont très rares et impliquent une autorisation expresse du maître ${ }^{254}$. La plus fréquemment envisagée est celle où un apprenti, dans l'impossibilité de poursuivre sa formation chez son maittre, peut la parachever ailleurs ${ }^{25}$. En général les brevets prévoient leur résiliation de plein droit en cas d'impossibilité de poursuite de l'apprentissage du fait d'une incapacité physique de l'apprenti ${ }^{256}$ ou du maitre, ce qui autorise l'apprenti à finir sa formation ailleurs. Enfin, un cas plutôt exceptionnel mérite d'être cité. Le brevet signé le 2 novembre 1784, par lequel François Baxès maçon habitant à Maureillas place son fils André en apprentissage chez Pierre Cabanes tisserand à lin du même village aux termes duquel « lesdites parties ont convenu que des que ledit apprentif sera en état de travailler il luy sera libre et permis de faire la toile et autres choses qui regardent le métier de tixeran que ledit son père voudra faire faire et ce avec les outils dudit son maitre et sans payer la moindre chose à ce dernier et sans luy retourner aucun jour sous la condition cependant que ledit André Baxès travaillant pour ledit son père ledit Cabanes ne sera nullement obligé à luy fournir les susdits aliments $»^{257}$. L'apprenti est donc libre de travailler sur ordre

250 - 3E 56/160, nं 120, brevet rédigé par Joseph Galard le 24 avril 1783.

251 - Quinze brevets comportent ce délai, notamment le brevet du 9 mars 1789 (3E 5/86, $n^{\circ} 117$ ).

252 - Trente-quatre brevets prévoient ce délai, notamment le brevet du 12 juillet 1780 (3E 5/76, n 310).

253 - En Poitou, certains statuts autorisent l'apprenti à revenir à l'atelier pendant un an, voir P. BOISSONNADE, op. cit., p. 5l, n. 5. A Bordeaux, les délais sont généralement bien plus courts, notamment dans les métiers où la main-d'œuvre est abondante et où peu de qualification est nécessaire. En revanche les arquebusiers autorisent l'apprenti à revenir pendant un délai de trois mois, voire davantage si le maître y consent. B. GALLINATO, op. cit., p. 98.

254 - Un brevet du 20 mai 1780 plaçant Jean Serdanne en apprentissage chez Joseph Marti dit Garriga maitre cordier à Ria énonce " qu'il ne sera nullement permis audit Serdanne de travailler en aucune boutique du présent ressort sans l'expresse permission dudit Marti » (3E 19/1062, n’ 46 de la « 3e main »).

255 - 3E 5/76, n 165, brevet du 30 mars 1780.

256 - 3E 5/78, nं210, brevet du 17 juin 1781. Ce brevet prévoit le cas « de rhumatisme et toute autre maladie ou incommodité ». En outre, un acte de résiliation du 23 avril 1783 (3E 9/391) énonce que Joseph Ramonatxo apprenti souffre de diverses « incommodités qui l'empêcheraient de pouvoir continuer son apprentissage » selon son maitre qui, en conséquence, demande à résilier le brevet conclu le ler novembre 1782 (3E 9/390).

257 - $3 E$ 44/65, nं192. 
de son père avec les outils de son maître qui est alors libéré de son obligation de le nourrir.

Plutôt que de prendre un garçon à la place de l'apprenti absent, le maître peut parfois préférer "se faire rembourser le montant du coût de ses aliments et habillement $" 258$. Sinon le brevet conclu le 18 juillet 1786 au profit de Marc Briu par sa mère précise qu'en cas de fuite de son fils, si celle-ci ne parvient pas à le faire " revenir sous quinzaine à son devoir ", le maître " pourra exiger la somme de cinquante livres desdits mère et fils Briu pour l'indemniser du travail qu'il pourroit faire ${ }^{259}$. C'est une sorte de rachat du temps restant à accomplir à l'apprenti ${ }^{260}$. Peu de brevets ${ }^{261}$ envisagent de sanctionner l'apprenti ou sa famille par une clause pénale ${ }^{262}$ ou une " amende $»^{263}$.

Au terme de cette analyse, il ressort que l'apprentissage " formait essentiellement " la clé de voûte " de l'édifice corporatif d'Ancien Régime où, malgré des abus et parfois des circonstances défavorables, vécut si longtemps et prospéra tout le peuple des maîtres et artisans, le monde du travail de l'ancienne France ${ }^{264}$. En effet, de manière presque naturelle, si l'on excepte les prescriptions des statuts professionnels, un équilibre contractuel s'établissait entre maitre et apprenti au regard de divers paramètres tels que le prix, la durée, les conditions de l'entretien de l'apprenti ou encore le salaire à lui verser afin d'ajuster le contrat en fonction des aspirations et moyens des parties en présence. Il est probable que la pratique des actes notariés permettait de modérer « les tentations d'excès des maîtres d'apprentissage ${ }^{265}$. Ainsi le lien de subordination s'en trouvait noyé dans les obligations réciproques des parties qui reposaient sur une certaine confiance. Un notaire qualifie d'ailleurs le départ définitif de l'apprenti de « dézertion » dans les quatre brevets qu'il rédige pour cette période ${ }^{266}$. Antoine Lavall, notaire à Prades, insère quant à lui, dans ses six brevets une clause aux termes de laquelle ceux qui placent l'apprenti assurent qu'il est fidèle ${ }^{267}$. A l'opposé certains notaires usent d'un vo-

258 - 3E 10/104, $f^{\circ} 397$ r ${ }^{\circ}$, brevet du 15 août 1781.

259 - 3 E 14/71, n 451.

260 - B. Gallinato, op. cit., p. 103.

261 - Rachel Rodriguez relève des sommes à payer en cas d'inexécution du brevet s'élevant généralement à 25 et pouvant atteindre 100 livres. R. RODRIGUEZ, op. cit., p. 49.

262 - J. DASSONVILLE, op. cit., p. 43.

263 - Elle est pratiquée dans quelques contrats en Bourgogne aux XIVe et XVe siècles, à Troyes au XVIle siècle, à Bordeaux au XVIIIle siècle et prévue dans certains statuts de métiers parisiens. P. DIDIER, op. cit., p. 42 ; L. MorIN, op. cit., p. 18 ; B. Gallinato, op. cit., p. 99 ; J. De BONNE, op. cit., p. 39.

264 - J. DE BONNE, op. cit., p. 54.

265 - A.-M. COCULA, op. cit., p. 431.

266 - Il s'agit de Joseph Nicolau-Coromina, notaire à Villefranche-de-Conflent dans les brevets des 20 mai 1780, 21 mai 1782,3 et 8 juin 1788 (3E 19/1062, n 46 de la « 3 e main », 3E 24/51, n 43 et 3E 24/54, nos 38 et 40).

267 - Cinq brevets utilisent une formule similaire (voir par exemple le brevet du 29 mai 1780, 3E 21/435, n 72, $f^{\circ} 145$ ). Le sixième est différent parce que l'apprenti se plaçant lui-même affirme donc « être fidelle » $\left(3 E 21 / 435, n^{\circ} 29, f^{\circ} 59\right.$, brevet du 14 février 1780). Louis Morin donne l'exemple d'un curé, grand-oncle d'un enfant qu'il place en apprentissage se portant garant et responsable de sa fidélité. L. MorIN, op. cit., p. 14. 
cabulaire carcéral. Joseph Gauze, notaire à Rivesaltes évoque la possible évasion d'un apprenti. A titre d'exemple, le 9 septembre 1783 il dresse un brevet plaçant Joseph Sarda chez Pierre Poujol cordonnier à Opoul dans lequel il prévoit que "si ledit Sarda sevade de la maison de son maitre $»^{268}$ ce dernier pourra prendre un garçon à sa place. Il est vrai que parfois la mise en apprentissage "s'effectue sans la volonté de l'enfant, parfois contre cette volonté [et] ressemble à une vente temporaire de la personne ${ }^{269}$. Mais «il faut se souvenir que les mœurs d'autrefois étaient grossières, et qu’à tout prendre le milieu familial où l'apprenti grandissait était très propre à lui inspirer l'esprit de corps, à lui donner une forte instruction technique, à le préparer en un mot au rôle de compagnon ou de maître ${ }^{270}$. Pour autant le brevet n'était pas un moyen d'exploiter la main d'œuvre juvénile car dans ce type de contrat où joue indéniablement l'intuitu personae, "obligations morales et professionnelles sont inséparables pour l'un et pour l'autre $»^{271}$. A l'instar d'autres institutions, religieuses, familiales ou professionnelles, l'apprentissage apparaît comme un rituel de passage car au terme prévu, «la première période de la vie laborieuse de l'enfant est terminée ; demain, il sera un homme ${ }^{272}$ aspirant à devenir l'égal de son maitre et de son père.

268 - 3E 36/109, n 122.

269 - J. DASSONVILLE, op. cit., p. 45.

270 - P. BOISSONNADE, op. cit., p. 52.

271 - A.-M. CoCULA, op. cit., p. 430.

272 - L. MORIN, op. cit., p. 20. 